\title{
Higgs, di-Higgs and tri-Higgs production via SUSY processes at the LHC with $14 \mathrm{TeV}$
}

\author{
Melissa van Beekveld, ${ }^{a, b}$ Wim Beenakker, ${ }^{a, b, c}$ Sascha Caron, ${ }^{a, b}$ Remco Castelijn, ${ }^{a, b}$ \\ Marie Lanfermann ${ }^{a, b}$ and Antonia Struebig ${ }^{a, b}$ \\ ${ }^{a}$ Institute for Mathematics, Astrophysics and Particle Physics, Faculty of Science, \\ Radboud University Nijmegen, \\ Mailbox 79, P.O. Box 9010, NL-6500 GL Nijmegen, The Netherlands \\ ${ }^{b}$ Nikhef, \\ Science Park, Amsterdam, The Netherlands \\ ${ }^{c}$ Institute of Physics, University of Amsterdam, \\ Science Park 904, 1018 XE Amsterdam, The Netherlands \\ E-mail: mcbeekveld@gmail.com, W.Beenakker@science.ru.nl, \\ scaron@cern.ch, r.castelijn@nikhef.nl, marie.lanfermann@gmail.com, \\ antonia.struebig@cern.ch
}

ABSTRACT: We have systematically investigated the production of a Higgs boson with a mass of about $125 \mathrm{GeV}$ in the decays of supersymmetric particles within the phenomenological MSSM (pMSSM). We find regions of parameter space that are consistent with all world data and that predict a sizeable rate of anomalous Higgs, di-Higgs and even tri-Higgs events at the $14 \mathrm{TeV}$ LHC. All relevant SUSY production processes are investigated. We find that Higgs bosons can be produced in a large variety of SUSY processes, resulting in a large range of different detector signatures containing missing transverse momentum. Such Higgs events are outstanding signatures for new physics already for the early $14 \mathrm{TeV}$ LHC data. SUSY processes are also important to interprete deviations found in upcoming Standard Model Higgs and di-Higgs production measurements.

KeYwords: Supersymmetry Phenomenology

ArXiv EPrint: 1501.02145v2 


\section{Contents}

1 Introduction 1

2 Supersymmetric decays into the lightest Higgs 2

2.1 Neutralino and chargino decays into the lightest Higgs 4

2.2 Sfermion decays into the lightest Higgs 8

2.3 Heavy Higgs-boson decays into the lightest Higgs 9

3 Finding candidate pMSSM models: simulation and constraints $\quad 10$

$\begin{array}{lll}3.1 & \text { Generation and pre-selection of pMSSM model-sets } & 10\end{array}$

$\begin{array}{lll}3.2 & \text { Parameter space coverage with a particle filter } & 11\end{array}$

$\begin{array}{lll}3.3 & \text { Experimental constraints } & 11\end{array}$

3.4 ATLAS constraints: event generation, fast simulation and analysis $\quad 14$

4 Analysis of the candidate models $\quad 17$

$\begin{array}{lll}4.1 & \text { Branching ratios } & 17\end{array}$

$\begin{array}{lll}4.2 & \text { Event generation, fast simulation and analysis } & 17\end{array}$

4.3 Determining the expected number of events with Higgs bosons 19

$\begin{array}{lll}\text { 4.3.1 Higgs production via SUSY processes } & 19\end{array}$

$\begin{array}{lll}\text { 4.3.2 } & \text { Di-Higgs production via SUSY processes } & 22\end{array}$

$\begin{array}{lll}\text { 4.3.3 Tri-Higgs production via SUSY processes } & 24\end{array}$

4.4 The lightest Higgs boson from heavy-Higgs production processes 24

4.5 Kinematic distributions for Higgs events from SUSY 27

5 Conclusion $\quad 33$

A Selected illustrative figures $\quad 34$

\section{Introduction}

The Higgs-boson discovery at the Large Hadron Collider (LHC) [1, 2] marks the beginning of a new era in particle physics. It gives us exciting new possibilities to study the physics of the Standard Model (SM) of particle physics. In this paper we investigate the next level of Higgs-boson searches, namely the possibility that Higgs bosons with a mass of about $125 \mathrm{GeV}$ are produced by processes involving physics beyond the SM.

Supersymmetry (SUSY) [3-16] is one of the conceivable extensions of the SM. It could provide a natural candidate for cold dark matter if R-parity is conserved [17, 18] and it allows for a stabilization of the electroweak scale by reducing the fine tuning of higherorder corrections to the Higgs mass [16, 19-23]. In its minimal version, i.e. the Minimal Supersymmetric Standard Model (MSSM), SUSY predicts superpartners for the existing 
SM particles and two Higgs doublets instead of one. On top of that, R-parity is assumed to be conserved in the MSSM, which results in the existence of a lightest supersymmetric particle (LSP). If the LSP is a neutralino, i.e. a Majorana-fermion superpartner associated with the neutral SM bosons in the electroweak sector, it is only weakly interacting and stable. It escapes detection, which results in missing transverse momentum in the detector.

In the present study we investigate systematically the possibilities to produce Higgs bosons with a mass $m_{h^{0}} \approx 125 \mathrm{GeV}^{1}$ in the decay of SUSY particles. This analysis is based on the phenomenological MSSM (pMSSM) [24, 25]. The pMSSM is scanned for parameter regions where the SUSY particles have a viable branching ratio to Higgs bosons. Only those models are selected that fulfil the current constraints on SUSY. The relevance for the upcoming LHC runs at $14 \mathrm{TeV}$ is discussed in detail and the most relevant Higgs production processes are identified. Higgs production via particular SUSY processes has been studied e.g. in [26-29]. We calculate the allowed production rates for anomalous Higgs, di-Higgs and tri-Higgs events. Subsequently, LHC events are simulated for each interesting model. These events are classified into topologies according to the SM particles produced in association with the Higgs boson(s) and the Higgs kinematics is studied. We identify topologies that are interesting for extending the current SUSY searches. Experimentally the events might be best detectable by explicitly "tagging" the Higgs boson(s) in SUSY searches. Since the invariant mass of the (lightest) Higgs boson is known and well reconstructable in many decay modes, and since we know that the SM rate to produce Higgs events with large missing transverse momentum (and maybe other SM particles) is small, a "Higgs-tag" can provide a unique signature for new physics. A special "Higgs-tag" for a boosted Higgs has also been suggested [30-33].

A few analyses have already searched for such events in ATLAS and CMS data. Higgs production via $\widetilde{\chi}_{2}^{0} \widetilde{\chi}_{1}^{ \pm}$neutralino-chargino production has been investigated in ATLAS [34] and CMS [35, 36]. In addition, searches have been pursued by CMS for a simplified model with a Higgs produced in top squark decays $[37,38]$. The present study aims to systematically investigate the possibility to produce Higgs bosons within the current constraints on SUSY by considering all relevant SUSY processes and decays.

This paper is organized as follows. In section 2 the most important supersymmetric decay mechanisms for producing light Higgs bosons are discussed. In section 3 the pMSSM parameter space is scanned for models that are consistent with all current experimental constraints on SUSY and that have the potential to produce sizeable Higgs-boson event rates. Finally, in section 4 the surviving pMSSM models are studied with regard to the expected Higgs-boson event rates at the early stages of the upcoming LHC run and with regard to special kinematical features, such as boosts and missing transverse momentum.

\section{Supersymmetric decays into the lightest Higgs}

In view of its important role in producing Higgs bosons, we start with a detailed discussion of the neutralino/chargino sector. In the MSSM some of the superfields mix as a result of SUSY breaking to form new mass eigenstates. Let's first consider the neutral SM bosons in

\footnotetext{
${ }^{1}$ This study assumes that the discovered Higgs boson at $m_{h^{0}} \approx 125 \mathrm{GeV}$ is the lightest neutral CP-even Higgs boson of the MSSM.
} 
the electroweak sector, i.e. the hypercharge $B$ boson, neutral weak $W^{3}$ boson and neutral components of the two Higgs doublets. The associated Majorana-fermion superpartners, i.e. the Bino $\widetilde{B}$, neutral Wino $\widetilde{W}^{3}$ and neutral Higgsinos $\widetilde{H}_{d}^{0}$ and $\widetilde{H}_{u}^{0}$, mix to form neutral mass eigenstates called neutralinos $\left(\widetilde{\chi}_{1,2,3,4}^{0}\right.$, numbered in increasing mass order). This mixing is caused by off-diagonal terms in the neutralino mass matrix, which acts on the Bino, Wino and Higgsino fields [39]:

$$
\mathcal{L}_{\text {neutralino mass }}=-\frac{1}{2}\left(\psi^{0}\right)^{T} M_{\widetilde{\chi}^{0}} \psi^{0}+\text { h.c. },
$$

where

$$
\psi^{0}=\left(\begin{array}{c}
\widetilde{B} \\
\widetilde{W}^{3} \\
\widetilde{H}_{d}^{0} \\
\widetilde{H}_{u}^{0}
\end{array}\right)
$$

and

$$
M_{\widetilde{\chi}^{0}}=\left(\begin{array}{cccc}
M_{1} & 0 & -c_{\beta} s_{\theta_{W}} m_{Z} & s_{\beta} s_{\theta_{W}} m_{Z} \\
0 & M_{2} & c_{\beta} c_{\theta_{W}} m_{Z} & -s_{\beta} c_{\theta_{W}} m_{Z} \\
-c_{\beta} s_{\theta_{W}} m_{Z} & c_{\beta} c_{\theta_{W}} m_{Z} & 0 & -\mu \\
s_{\beta} s_{\theta_{W}} m_{Z} & -s_{\beta} c_{\theta_{W}} m_{Z} & -\mu & 0
\end{array}\right) .
$$

Here $s_{\alpha} \equiv \sin \alpha$ and $c_{\alpha} \equiv \cos \alpha$. The parameters $M_{1}$ and $M_{2}$ are the SUSY-breaking mass parameters for the Bino and Winos, $\mu$ is the SUSY version of the SM Higgs-mass parameter, $\cos \theta_{W}=m_{W} / m_{Z}$ is the ratio of the SM $W$-boson and $Z$-boson masses, and $\tan \beta$ is the ratio of the two Higgs vacuum expectation values.

A similar mixing phenomenon occurs in the charged sector, belonging to the charged weak bosons $W^{ \pm}$and the charged components of the Higgs doublets. The associated Dirac-fermion superpartners, i.e. the charged Winos $\widetilde{W}^{ \pm}$and Higgsinos $\widetilde{H}_{u / d}^{ \pm}$, mix to form charged mass eigenstates called charginos $\left(\widetilde{\chi}_{1,2}^{ \pm}\right.$, numbered in increasing mass order $)$as a result of the mixing in the chargino mass matrix [39]:

$$
M_{\widetilde{\chi}^{ \pm}}=\left(\begin{array}{cc}
M_{2} & \sqrt{2} c_{\beta} c_{\theta_{W}} m_{Z} \\
\sqrt{2} s_{\beta} c_{\theta_{W}} m_{Z} & \mu
\end{array}\right) .
$$

The mixing in the neutralino and chargino mass matrices stems from terms that go with the $Z$-boson mass. However, in the case that $M_{1}, M_{2}$ and $|\mu|$ largely exceed the mass of the $Z$-boson, the mixing terms are relatively small. If we neglect the mixing terms, the neutralinos are either a Bino, a Wino or a symmetric/antisymmetric mix of both Higgsino states, $\widetilde{H}_{S / A}^{0} \equiv \frac{1}{\sqrt{2}}\left(\widetilde{H}_{u}^{0+} /-\widetilde{H}_{d}^{0}\right)$. The charginos are in that case either a charged Wino or a charged Higgsino. The composition for all possible regimes is shown in table 1. In this simplified case the mass of the Bino neutralino is $M_{1}$, the masses of the Wino neutralino and charginos are $M_{2}$, and the masses of the Higgsino neutralinos and charginos are $|\mu|$.

In fact some of the eigenvalues of the mass matrices will turn out to be negative. For instance, $\widetilde{H}_{S}^{0}$ corresponds to the eigenvalue $-\mu$, whereas $\widetilde{H}_{A}^{0}$ corresponds to the oppositesign eigenvalue $+\mu$. In order to arrive at a proper (non-negative) definition of the mass of 


\begin{tabular}{|ccc|}
\hline Regime & Composition neutralinos & Composition charginos \\
\hline$M_{1}<M_{2}<|\mu|$ & $(\widetilde{B}, \widetilde{W}, \widetilde{H}, \widetilde{H})$ & $(\widetilde{W}, \widetilde{H})$ \\
$M_{1}<|\mu|<M_{2}$ & $(\widetilde{B}, \widetilde{H}, \widetilde{H}, \widetilde{W})$ & $(\widetilde{H}, \widetilde{W})$ \\
$|\mu|<M_{1}<M_{2}$ & $(\widetilde{H}, \widetilde{H}, \widetilde{B}, \widetilde{W})$ & $(\widetilde{H}, \widetilde{W})$ \\
$|\mu|<M_{2}<M_{1}$ & $(\widetilde{H}, \widetilde{H}, \widetilde{W}, \widetilde{B})$ & $(\widetilde{H}, \widetilde{W})$ \\
$M_{2}<|\mu|<M_{1}$ & $(\widetilde{W}, \widetilde{H}, \widetilde{H}, \widetilde{B})$ & $(\widetilde{W}, \widetilde{H})$ \\
$M_{2}<M_{1}<|\mu|$ & $(\widetilde{W}, \widetilde{B}, \widetilde{H}, \widetilde{H})$ & $(\widetilde{W}, \widetilde{H})$ \\
\hline
\end{tabular}

Table 1. Composition of the neutralinos $\left(\widetilde{\chi}_{1}^{0}, \widetilde{\chi}_{2}^{0}, \widetilde{\chi}_{3}^{0}, \widetilde{\chi}_{4}^{0}\right)$ and charginos $\left(\widetilde{\chi}_{1}^{ \pm}, \widetilde{\chi}_{2}^{ \pm}\right)$.

all particles, an extra factor $\gamma^{5}$ will have to be absorbed into the definition of the negativemass eigenstates, which flips the sign of the corresponding mass eigenvalue. As we will see, this extra factor $\gamma^{5}$ has important consequences for the decay properties of the neutralinos.

When we switch on the mixing again, mixed neutralino states consisting of Binos, Winos and Higgsinos will exist. However, since the mixing is small, there will always be a part that dominates the state, which we then refer to as Binolike, Winolike or Higgsinolike. The true masses of all the neutralinos and charginos behave as in the previously discussed simplified case, which is governed by the three mass parameters $M_{1}, M_{2}$ and $\mu$.

\subsection{Neutralino and chargino decays into the lightest Higgs}

If we choose the lightest neutralino to be the LSP, all supersymmetric particles will eventually decay into a lightest neutralino. The branching ratios of the most important direct decay channels of neutralinos into the lightest Higgs boson $h^{0}$ accompanied by a LSP are shown in figure 1. The lightest chargino plays an important role if it is of almost the same mass as the lightest neutralino. Therefore the branching ratio of figure $1 \mathrm{~d}$ is also included.

Some of the features of these decay processes can be explained very well kinematically with the previously discussed simplified case. For example, the decay $\widetilde{\chi}_{2}^{0} \rightarrow \widetilde{\chi}_{1}^{0}+h^{0}$ is very unlikely in the case that $M_{1}, M_{2}>|\mu|$ or when the smallest two parameters of the set $M_{1}$, $M_{2},|\mu|$ are relatively close (i.e. less than $m_{h^{0}}$ apart), as can be seen in figure 1a. This is because both neutralinos have more or less the same mass in that case, which means that the decay $\widetilde{\chi}_{2}^{0} \rightarrow \widetilde{\chi}_{1}^{0}+h^{0}$ is kinematically not allowed. For the same reason the decay $\tilde{\chi}_{2}^{ \pm} \rightarrow \widetilde{\chi}_{1}^{ \pm}+h^{0}$ is greatly suppressed in the region around $M_{2} \approx|\mu|$, as can be seen in figure $1 \mathrm{~d}$. In figure $1 \mathrm{~b}$ we see that a similar thing holds for the decay $\widetilde{\chi}_{3}^{0} \rightarrow \widetilde{\chi}_{1}^{0}+h^{0}$ for $M_{2} \approx|\mu|<M_{1}$ or $M_{1} \approx|\mu|<M_{2}$, since in that case the lightest three neutralinos have more or less the same mass.

For some of the features of these decay processes, such as the apparent complementarity of $\operatorname{BR}\left(\widetilde{\chi}_{2}^{0} \rightarrow \widetilde{\chi}_{1}^{0}+h^{0}\right)$ and $\operatorname{BR}\left(\widetilde{\chi}_{3}^{0} \rightarrow \widetilde{\chi}_{1}^{0}+h^{0}\right)$ for $M_{2}>|\mu|>M_{1}$, we have to dig a little bit deeper. In order to facilitate the discussion we first list in table 2 the possible interactions between the Binos, Winos and Higgsinos, from which the neutralinos and charginos inherit their decay properties. In order to identify the interactions that involve the light Higgs boson, the two Higgs doublets are represented by the associated five Higgs mass eigenstates 


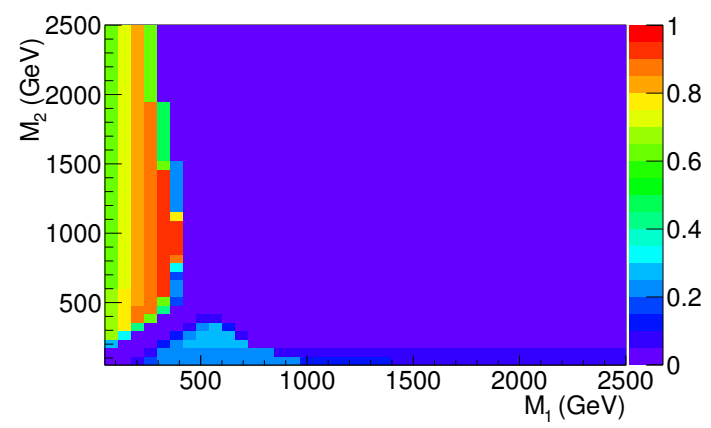

(a) $\operatorname{BR}\left(\widetilde{\chi}_{2}^{0} \rightarrow \widetilde{\chi}_{1}^{0}+h^{0}\right)$

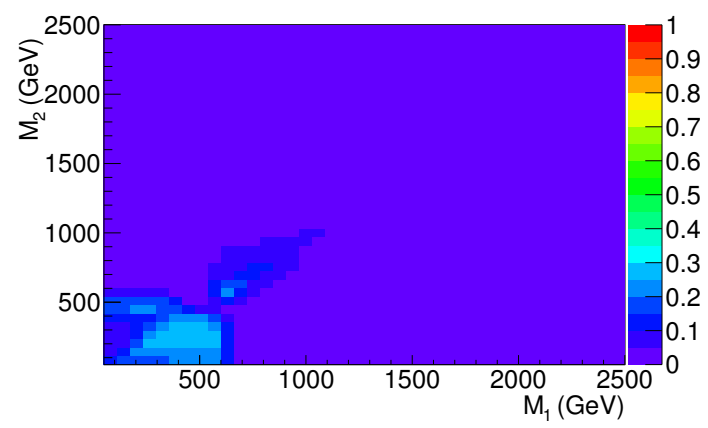

(c) $\operatorname{BR}\left(\widetilde{\chi}_{4}^{0} \rightarrow \widetilde{\chi}_{1}^{0}+h^{0}\right)$

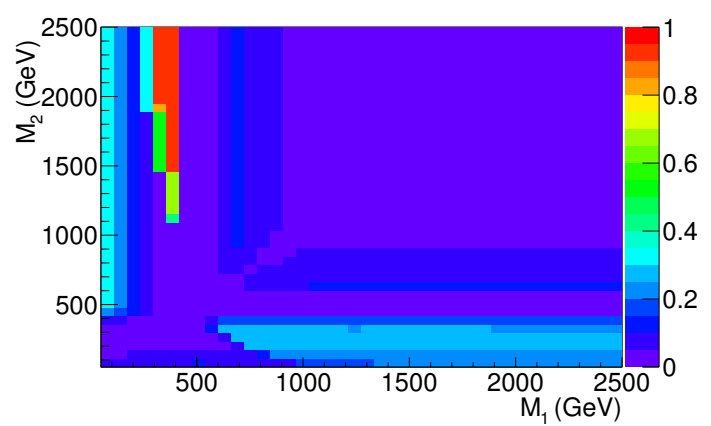

(b) $\operatorname{BR}\left(\widetilde{\chi}_{3}^{0} \rightarrow \widetilde{\chi}_{1}^{0}+h^{0}\right)$

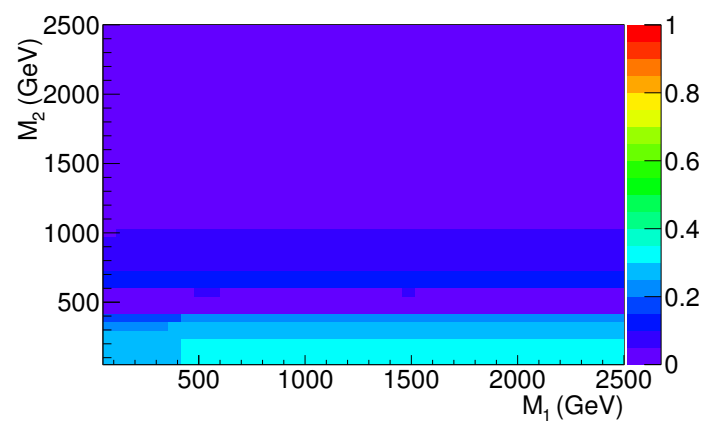

(d) $\operatorname{BR}\left(\widetilde{\chi}_{2}^{ \pm} \rightarrow \widetilde{\chi}_{1}^{ \pm}+h^{0}\right)$

Figure 1. Prominent direct branching ratios for the decay of neutralinos/charginos into the lightest Higgs boson and the lightest neutralino/chargino in the case that $\mu=500 \mathrm{GeV}, \tan \beta=50$ and all other parameters scaled up to very high values.

\begin{tabular}{|c||c|c|c|c|c|c|}
\hline & $\widetilde{B}$ & $\widetilde{W}^{3}$ & $\widetilde{H}_{S}^{0}$ & $\widetilde{H}_{A}^{0}$ & $\widetilde{W}^{ \pm}$ & $\widetilde{H}_{u / d}^{ \pm}$ \\
\hline$\widetilde{B}$ & & & $h^{0}, H^{0}, A^{0}$ & $h^{0}, H^{0}, A^{0}$ & & $H^{\mp}$ \\
$\widetilde{W}^{3}$ & & & $h^{0}, H^{0}, A^{0}$ & $h^{0}, H^{0}, A^{0}$ & $W^{\mp}$ & $H^{\mp}$ \\
$\widetilde{H}_{S}^{0}$ & $h^{0}, H^{0}, A^{0}$ & $h^{0}, H^{0}, A^{0}$ & & $Z$ & $H^{\mp}$ & $W^{\mp}$ \\
$\widetilde{H}_{A}^{0}$ & $h^{0}, H^{0}, A^{0}$ & $h^{0}, H^{0}, A^{0}$ & $Z$ & & $H^{\mp}$ & $W^{\mp}$ \\
$\widetilde{W}^{ \pm}$ & & $W^{\mp}$ & $H^{\mp}$ & $H^{\mp}$ & $Z$ & $h^{0}, H^{0}, A^{0}$ \\
$\widetilde{H}_{u / d}^{ \pm}$ & $H^{\mp}$ & $H^{\mp}$ & $W^{\mp}$ & $W^{\mp}$ & $h^{0}, H^{0}, A^{0}$ & $Z$ \\
\hline
\end{tabular}

Table 2. Interactions between the Binos, Winos and Higgsinos. The entries indicate which fields are involved in the interaction.

$h^{0}, H^{0}, A^{0}$ and $H^{ \pm}$. The $h^{0}$ field corresponds to the light CP-even Higgs boson, whereas the other four fields correspond to the heavy CP-even, CP-odd and charged Higgs bosons, respectively. 
Besides the kinematical observations mentioned before, we observe the following features in the various mass domains. These features mainly involve the competition between the decay modes into the $h^{0}$ and alternative decay modes involving $W$ or $Z$ bosons.

The Binolike-Winolike-Higgsinolike mass domain $M_{1}<M_{2}<|\mu|$ :

- The decay $\widetilde{\chi}_{2}^{0} \rightarrow \widetilde{\chi}_{1}^{0}+h^{0}$ tends to dominate the branching ratio of $\widetilde{\chi}_{2}^{0}$ if it is kinematically allowed, resulting in values for the branching ratio $\operatorname{BR}\left(\widetilde{\chi}_{2}^{0} \rightarrow \widetilde{\chi}_{1}^{0}+h^{0}\right)$ that can get close to unity (see figure 1a). As can be read off from table 2 , this is caused by the fact that the decay into the $h^{0}$ involves the (suppressed) Higgsino component of either the Binolike $\widetilde{\chi}_{1}^{0}$ or Winolike $\widetilde{\chi}_{2}^{0}$, whereas the decay into a $Z$ boson involves the (double suppressed) Higgsino components of both these neutralinos. At the same time the decay $\widetilde{\chi}_{2}^{0} \rightarrow \widetilde{\chi}_{1}^{ \pm}+W^{\mp}$ is not allowed kinematically since $m_{\widetilde{\chi}_{1}^{ \pm}} \approx m_{\widetilde{\chi}_{2}^{0}}$.

- The branching ratios $\operatorname{BR}\left(\widetilde{\chi}_{3,4}^{0} \rightarrow \widetilde{\chi}_{1}^{0}+h^{0}\right)$ can only reach values that are substantially smaller (see figures $1 \mathrm{~b}$ and $1 \mathrm{c}$ ), since the alternative decay modes $\widetilde{\chi}_{3,4}^{0} \rightarrow \widetilde{\chi}_{1}^{ \pm}+W^{\mp}$ and $\tilde{\chi}_{3,4}^{0} \rightarrow \widetilde{\chi}_{2}^{0}+h^{0} / Z$ seriously reduce the maximum branching ratio for direct decays into the LSP. Note, however, that the $\widetilde{\chi}_{3,4}^{0}$ neutralinos might be of interest for di-Higgs decay modes in view of the possible two-step decays $\widetilde{\chi}_{3,4}^{0} \rightarrow \widetilde{\chi}_{2}^{0}+h^{0}$ followed by $\widetilde{\chi}_{2}^{0} \rightarrow \widetilde{\chi}_{1}^{0}+h^{0}$.

The Winolike-Binolike-Higgsinolike mass domain $M_{2}<M_{1}<|\mu|$ : the same arguments in principle apply to this mass domain. However, in this case the (single suppressed) decay mode $\widetilde{\chi}_{2}^{0} \rightarrow \widetilde{\chi}_{1}^{ \pm}+W^{\mp}$ cannot be avoided since $m_{\widetilde{\chi}_{1}^{ \pm}} \approx m_{\widetilde{\chi}_{1}^{0}}$. As a result $\operatorname{BR}\left(\widetilde{\chi}_{2}^{0} \rightarrow \widetilde{\chi}_{1}^{0}+h^{0}\right)$ will at most reach 0.3 in this mass regime.

The Binolike-Higgsinolike-Winolike mass domain $M_{1}<|\mu|<M_{2}$ :

- We observe both very large (almost unity) and very small branching ratios for the decay into the $h^{0}$, with as additional striking feature the apparent complementarity of $\operatorname{BR}\left(\widetilde{\chi}_{2}^{0} \rightarrow \widetilde{\chi}_{1}^{0}+h^{0}\right)$ and $\operatorname{BR}\left(\widetilde{\chi}_{3}^{0} \rightarrow \widetilde{\chi}_{1}^{0}+h^{0}\right)$ (see figures 1a and $1 \mathrm{~b}$ ). This has to do with the occurrence of negative-mass eigenstates in the Higgsino sector and the associated factor $\gamma^{5}$ that is introduced in order to flip the sign of the mass eigenvalue. If $\widetilde{\chi}_{2}^{0}$ corresponds to a genuine positive-mass eigentstate, then $\widetilde{\chi}_{2}^{0} \rightarrow \widetilde{\chi}_{1}^{0}+h^{0}$ is an unsuppressed scalar decay mode (see table 2) that tends to dominate the single suppressed decay into a $Z$ boson. If $\widetilde{\chi}_{2}^{0}$ corresponds to a negative-mass eigenstate, then $\widetilde{\chi}_{2}^{0} \rightarrow \widetilde{\chi}_{1}^{0}+h^{0}$ is a velocity-suppressed pseudoscalar decay mode and this time the decay mode into a $Z$ boson dominates. The observed complementarity follows from the fact that $\widetilde{\chi}_{2}^{0}$ and $\widetilde{\chi}_{3}^{0}$ correspond to opposite-sign mass eigenvalues, while at the same time the suppression factors are such that the role of $h^{0}$ and $Z$ are effectively interchanged in the two cases [40].

- The branching ratio $\mathrm{BR}\left(\widetilde{\chi}_{4}^{0} \rightarrow \widetilde{\chi}_{1}^{0}+h^{0}\right)$ is even more reduced than in the previously discussed mass domains (see figure 1c). This is caused by the larger number of competing alternative decay modes, i.e. $\widetilde{\chi}_{4}^{0} \rightarrow \widetilde{\chi}_{1}^{ \pm}+W^{\mp}$ and $\widetilde{\chi}_{4}^{0} \rightarrow \widetilde{\chi}_{2,3}^{0}+h^{0} / Z$. Note, though, that the $\widetilde{\chi}_{4}^{0}$ neutralino might be of interest for di-Higgs decay modes in view of the possible two-step decays $\widetilde{\chi}_{4}^{0} \rightarrow \widetilde{\chi}_{2,3}^{0}+h^{0}$ followed by $\widetilde{\chi}_{2,3}^{0} \rightarrow \widetilde{\chi}_{1}^{0}+h^{0}$. 
The Winolike-Higgsinolike-Binolike mass domain $M_{2}<|\mu|<M_{1}$ : the previous arguments in principle apply to this mass domain as well. However, in this case the decay modes $\widetilde{\chi}_{2,3}^{0} \rightarrow \widetilde{\chi}_{1}^{ \pm}+W^{\mp}$ cannot be avoided. This reduces the maximum combined branching ratio for the decays into $h^{0}$ and $Z$ to roughly 0.3 . For this maximum combined branching ratio again a complementarity phenomenon is observed (see figures $1 \mathrm{a}$ and $1 \mathrm{~b}$ ).

The Higgsinolike LSP mass domain $|\mu|<M_{1,2}$ :

- As mentioned before, the decay $\widetilde{\chi}_{2}^{0} \rightarrow \widetilde{\chi}_{1}^{0}+h^{0}$ is not allowed kinematically since both neutralinos have more or less the same mass in the Higgsinolike LSP case.

- The branching ratio $\operatorname{BR}\left(\widetilde{\chi}_{3}^{0} \rightarrow \widetilde{\chi}_{1}^{0}+h^{0}\right)$ is strongly reduced, since the alternative decay modes $\widetilde{\chi}_{3}^{0} \rightarrow \widetilde{\chi}_{1}^{ \pm}+W^{\mp}$ and $\widetilde{\chi}_{3}^{0} \rightarrow \widetilde{\chi}_{2}^{0}+h^{0} / Z$ cannot be avoided as a result of the approximate mass-degeneracy of $\widetilde{\chi}_{1}^{0}, \widetilde{\chi}_{2}^{0}$ and $\widetilde{\chi}_{1}^{ \pm}$. Moreover, based on the abovegiven complementarity discussion we know that at least one of the $\widetilde{\chi}_{3}^{0} \rightarrow \widetilde{\chi}_{2}^{0}+h^{0} / Z$ decay modes will not be suppressed. Note, though, that $\widetilde{\chi}_{3}^{0} \rightarrow \widetilde{\chi}_{2}^{0}+h^{0}$ in itself already constitutes a direct SUSY decay channel into the lightest Higgs boson.

The chargino decays:

- The lightest chargino can never decay directly into the lightest Higgs boson. This is due to the fact that R-parity conservation forbids a decay to a neutral Higgs boson. For this a lighter charged supersymmetric fermion is needed, which is not present in the case of the lightest chargino. It is possible for a lightest chargino to decay into three particles, but such three-particle chargino decay modes with a Higgs boson featuring in the final state are rather rare.

- For $M_{2}<|\mu|$ the branching ratio $\operatorname{BR}\left(\tilde{\chi}_{2}^{ \pm} \rightarrow \tilde{\chi}_{1}^{ \pm}+h^{0}\right)$ can reach maximum values of up to 0.35 as a result of the competition from the unavoidable decay mode $\widetilde{\chi}_{2}^{ \pm} \rightarrow \widetilde{\chi}_{1}^{0}+W^{ \pm}$ as well as the decay mode $\widetilde{\chi}_{2}^{ \pm} \rightarrow \widetilde{\chi}_{1}^{ \pm}+Z$. The total branching ratio for multi-step decays into the lightest Higgs boson can substantially exceed 0.35 in view of the possibility of two-step decays of the form $\widetilde{\chi}_{2}^{ \pm} \rightarrow \widetilde{\chi}_{2}^{0}+W^{ \pm}$followed by $\widetilde{\chi}_{2}^{0} \rightarrow \widetilde{\chi}_{1}^{0}+h^{0}$ if $M_{1,2}<|\mu|$.

- For $|\mu|<M_{2}$ the branching ratio $\mathrm{BR}\left(\widetilde{\chi}_{2}^{ \pm} \rightarrow \widetilde{\chi}_{1}^{ \pm}+h^{0}\right)$ gets additionally reduced by the alternative decay mode to the second Higgsinolike neutralino. Again multi-step decay modes can substantially enhance the branching ratio for the decay into the $h^{0}$.

In conclusion, the branching ratios for direct decays of neutralinos/charginos into the LSP and the lightest Higgs boson can be pretty large, reaching maximum values close to one for $\widetilde{\chi}_{2,3}^{0}$. For $\widetilde{\chi}_{1}^{ \pm}$there is effectively no decay into the lightest Higgs boson. For the heavier states $\widetilde{\chi}_{4}^{0}$ and $\widetilde{\chi}_{2}^{ \pm}$the direct-decay branching ratios can reach 0.35 at best. However, for these heavy SUSY particles the total branching ratio for multi-step decays into the LSP and the lightest Higgs boson can be substantially larger if the non-Higgs decay step gives rise to $\widetilde{\chi}_{2,3}^{0}$, which can subsequently decay into the lightest Higgs boson with high probability. 


\subsection{Sfermion decays into the lightest Higgs}

Next we give a brief summary of the other supersymmetric decay channels that can produce a lightest Higgs boson, starting with the sfermions (squarks and sleptons), the scalar superpartners of the SM fermions (quarks and leptons). Such decay modes will play a role later on when the masses of the sfermions are not artificially scaled up to very high values. In this context it should also be noted that, apart from the interactions listed in table 2, the Binos, Winos and Higgsinos can also decay into fermion-sfermion pairs, involving the Yukawa interactions.

Since the Wino couples only to left-handed sfermions, the decays of left-handed $\left(\widetilde{f}_{L}\right)$ and right-handed $\left(\widetilde{f}_{R}\right)$ sfermions are different. In addition, the couplings to Higgsinos are Yukawa suppressed. This results in a profound difference between the decays of 1 st $/ 2$ nd generation sfermions and 3rd generation sfermions, since only the latter may have a large coupling to the Higgsinos.

Direct decays of sfermions into the lightest Higgs boson:

- First of all there is the possibility to have a mass difference between left- and righthanded sfermions. As a result, there is the possibility for $\widetilde{f}_{L, R} \rightarrow \widetilde{f}_{R, L}+h^{0}$ decay modes if the mass difference between the left- and right-handed sfermions exceeds $125 \mathrm{GeV}$. The couplings involved in this decay mode are Yukawa suppressed in the pMSSM. Therefore, the direct decay is mostly relevant for 3rd generation sfermions.

- The sfermions of the 3rd generation are mixtures of left- and right-handed states, indicated by $\widetilde{f}_{1,2}$ (numbered in increasing mass order). Therefore, there is an additional possibility for a direct decay via $\widetilde{f}_{2} \rightarrow \tilde{f}_{1}+h^{0}$. This decay can involve a non-Yukawa-suppressed (gauge) coupling between two left- or two right-handed components of the sfermion mass eigenstates. For 3rd generation squarks the gauge coupling and the Yukawa coupling can be of the same order of magnitude, which can lead to unexpected cancellations between both direct $h^{0}$ production mechanisms in that case.

Indirect decays of 1st/2nd generation sfermions into the lightest Higgs boson: sfermions can decay to heavy neutralinos or the heavy chargino, which can subsequently decay into lighter neutralinos or charginos and the lightest Higgs boson. The decay pattern differs for the left- and right-handed sfermions, depending on the composition of the LSP.

- Winolike LSP: the direct decay of the right-handed sfermions to the LSP is suppressed. If kinematically allowed the right-handed sfermions will decay to the Binolike neutralino, with the decay to the Higgsinolike states being Yukawa suppressed. As explained above, this Binolike neutralino can decay with a moderately large branching ratio to the $h^{0}$, since the decay to the $Z$ boson is double suppressed. The left-handed sfermions predominantly decay to the LSP, which strongly reduces indirect decays into the lightest Higgs boson.

- Higgsinolike LSP: the decay of the right and left-handed 1st/2nd generation sfermions to the LSP is Yukawa suppressed. If possible, these sfermions will decay to the heavier 
Bino- or Winolike states. As explained above, these states can decay with reduced branching fraction to the $h^{0}$.

- Binolike LSP: if the $\widetilde{\chi}_{1}^{0}$ is Binolike, the right-handed sfermions predominantly decay to the LSP. However, the left-handed sfermions still prefer to decay (if kinematically allowed) to the heavier Winolike neutralino/chargino. This is caused by an intrinsic $c_{\theta_{W}} / s_{\theta_{W}} \approx 1.9$ enhancement factor of the weak coupling of sleptons compared to the hypercharge coupling, with an additional factor 3 enhancement for squarks. As explained above, in these models the Winolike neutralino can have a large branching ratio to Higgs bosons. If it is a $\widetilde{\chi}_{4}^{0}$, then decays to charginos are also possible. If it is a $\widetilde{\chi}_{2}^{0}$, then its branching ratio to $h^{0}$ bosons is potentially very large and can be close to unity.

For 3rd generation sfermions the couplings to the Higgsinolike states are not Yukawa suppressed anymore and can even become large for top squarks. This results in a richer structure of possible decay modes and a more prominent role of Higgsinolike states as decay products. In that case indirect Higgs production can also become important in scenarios where Higgsinolike states have a large branching ratio into the lightest Higgs boson, as described in the previous subsection.

\subsection{Heavy Higgs-boson decays into the lightest Higgs}

Also the heavy Higgs particles can decay into the $h^{0}$. These particles are a consequence of SUSY, which requires more than one Higgs doublet, but as far as R-parity is concerned they qualify as "SM" particles. Consequently, these particles do not necessarily have to decay into the LSP and therefore do not necessarily give rise to large missing transverse momentum in their decay chains. A comprehensive overview of the decays of the heavy Higgs bosons is given in [41].

- Direct decays: as will be discussed later, all surviving MSSM models have $M_{A}$ values exceeding $300 \mathrm{GeV}$. This is known as the decoupling limit (large $M_{A}$ ) and consequently all heavy Higgs bosons have similar masses, which blocks decays among heavy Higgs bosons. The heavy CP-even Higgs boson $H^{0}$ can directly decay to two $h^{0}$ bosons. The CP-odd Higgs boson $A^{0}$ can decay to $h^{0} Z$. The charged Higgs bosons $H^{ \pm}$can decay to $W^{ \pm} h^{0}$. The corresponding branching ratios tend to be rather small, because in most surviving MSSM models $\tan \beta$ is relatively large $(>10)$ and consequently the decays of the heavy Higgs bosons to $b$-quarks are dominant. Later on we will encounter a couple of exceptional models that have the lowest values for $M_{A}$ and at the same time a relatively small value for $\tan \beta$ in order to survive the experimental constraints. Such models have noticeable branching ratios for direct heavy Higgs-boson decays into the lightest Higgs. More details can be found in ref. [41].

- Indirect decays: the heavy Higgs bosons also have the possibility to decay into heavy neutralinos or charginos (if kinematically allowed), especially if one of these decay states is Higgsinolike and the other Bino- or Winolike (see table 2). Those states can subsequently decay to $h^{0}$, sometimes with high branching ratios. This can, for instance, result in di-Higgs production from an $A^{0}$ decay. 


\section{Finding candidate pMSSM models: simulation and constraints}

The MSSM has more than 100 free parameters. Most of those parameters are not relevant for LHC physics. In the pMSSM the free parameters are reduced to 19 by demanding CP-conservation, minimal flavour violation and degenerate mass spectra for the 1st and 2nd generations of sfermions. The LSP is required to be the neutralino $\widetilde{\chi}_{1}^{0}$ in order to have a viable dark-matter candidate. This reduced model should cover a large fraction of the relevant SUSY phase space for $h^{0}$ production. The 19 remaining parameters are 10 sfermion masses, ${ }^{2} 3$ gaugino masses $M_{1,2,3}$, the ratio of the Higgs vacuum expectation values $\tan \beta$, the Higgsino mixing parameter $\mu$, the mass $m_{A}$ of the CP-odd Higgs-boson $A^{0}$ and 3 trilinear scalar couplings $A_{b, t, \tau}$.

\subsection{Generation and pre-selection of pMSSM model-sets}

SUSY-HIT [42] is used to generate the particle spectra of the 19-parameter pMSSM models. Only models are selected with a neutralino as LSP. The Higgs mass has been precisely determined by ATLAS and CMS to be 125.4 (ATLAS [43]) and $125.0 \mathrm{GeV}$ (CMS [44]) with uncertainties of $0.3-0.4 \mathrm{GeV}$ for each experiment. We select only models with a lightest Higgs boson $h^{0}$ within the range:

$$
124.4 \mathrm{GeV} \leq m_{h^{0}} \leq 126.5 \mathrm{GeV} .
$$

In addition we produce two statistically independent sets of models:

- Set A: Higgs production via direct decay of an arbitrary SUSY particle or a heavy Higgs boson.

As described in the previous section, Higgs production can occur via various different decays of SUSY particles. In addition, $h^{0}$ bosons can be produced in the decay of heavy Higgs bosons. For this set we require in the preselection that at least one SUSY particle or heavy Higgs boson has a direct branching ratio to $h^{0}$ exceeding $20 \%$.

- Set B: Higgs production via direct decays of charginos or neutralinos.

Since Higgs production via neutralino or chargino decays is most important, a second set of models dedicated to these decays is produced. For this set we required that at least one of the following direct branching ratios exceeds $20 \%$ :

$$
\begin{aligned}
\operatorname{BR}\left(\widetilde{\chi}_{2,3,4}^{0}\right. & \left.\rightarrow \widetilde{\chi}_{1}^{0}+h^{0}\right)>0.2 \quad \text { or } \\
\operatorname{BR}\left(\widetilde{\chi}_{2}^{ \pm}\right. & \left.\rightarrow \widetilde{\chi}_{1}^{ \pm}+h^{0}\right)>0.2 .
\end{aligned}
$$

The advantage of set B is that less model points are needed to study the most relevant Higgs production modes, since Higgs production predominantly originates from the decay of a heavy neutralino or chargino. Those neutralinos and charginos can be directly produced

\footnotetext{
${ }^{2}$ The corresponding sfermion labels are $\widetilde{Q}_{1}, \widetilde{Q}_{3}, \widetilde{L}_{1}, \widetilde{L}_{3}, \widetilde{u}_{1}, \widetilde{d}_{1}, \widetilde{u}_{3}, \widetilde{d}_{3}, \widetilde{e}_{1}$ and $\widetilde{e}_{3}$. Here 1 indicates the light-flavoured mass-degenerate 1st and 2nd generation sfermions and 3 the heavy-flavoured 3rd generation. The labels $\widetilde{Q}$ and $\widetilde{L}$ refer to the superpartners of the left-handed fermionic SU(2) doublets, whereas the other labels refer to the superpartners of the right-handed fermionic $\mathrm{SU}(2)$ singlets.
} 
or they are produced in cascade decays of predominantly squarks or gluinos, since these coloured SUSY particles can have a large cross section. The advantage of set A is its larger coverage of possible 3rd-generation and heavy-Higgs decay modes.

\subsection{Parameter space coverage with a particle filter}

This study has not the objective to provide a statistical interpretation like a "coverage" or a "likelihood" for a given parameter region. The objective is to find regions in the parameter space that are consistent with the global constraints on SUSY and where in addition the production of $h^{0}$ bosons is large (or close to maximal) in order to determine possible rates and topologies for SUSY Higgs production at the LHC. Each of our parameter sets represents a viable model point that could be realized in nature.

We use a simplified two-step particle filter algorithm [45] to find model points in the pMSSM parameter space.

1. First the 19 parameters of the pMSSM ( 3 gaugino masses, 6 squark masses, 4 slepton masses, 3 trilineair couplings, $M_{A}, \mu$ and $\tan \beta$ ) are chosen randomly from a flat prior distribution. The squark and slepton masses and $M_{A}$ have ranges between $100 \mathrm{GeV}$ and $3000 \mathrm{GeV}$. The Higgsino mixing term $\mu$, which in principle can be negative, ranges between $-3000 \mathrm{GeV}$ and $3000 \mathrm{GeV}$. This is also the case for the trilineair couplings, although we choose the ranges in that case between $-5000 \mathrm{GeV}$ and $5000 \mathrm{GeV}$ to be sure that the trilinear couplings will not restrict the simulation too much. The lower bound on the gaugino masses is chosen to be $10 \mathrm{GeV}$ to ensure that neutralinos, charginos and gluinos with very low masses are also evaluated. Finally, the ratio $\tan \beta$ of the Higgs vacuum expectation values is chosen between 1 and 50. For each set of pMSSM parameters SUSY-HIT [42] is used to generate the SUSY particle spectra and mixing matrices. Subsequently the preselection criteria of the previous subsection are checked. Model-sets are generated randomly within the given parameter range until we find 10000 model-sets fulfilling the preselection requirements.

2. These model-sets are then used as seeds (or particles) to build a posterior probability distribution from which further model-sets are generated. The posterior probability distribution is chosen as a sum of multi-dimensional Gaussian distributions centered around the parameter values $\vec{S}$ of each seed point. The multi-dimensional width of the Gaussian distributions is set to $10 \%, 25 \%$ and $40 \%$ of $\vec{L}_{d}$, where $L_{d}$ is the extent of the parameter space in dimension $d$ out of 19 . Around each seed further models are generated. The three sets are compared in order to evaluate the dependence on the width of the sampling. A comparison of the width dependence and a comparison of sets A and B is shown in figure 18 in the appendix. Since no significant difference is found all sets have been merged. This simulation process continues until in total at least 250000 models survive all preselection criteria.

\subsection{Experimental constraints}

The code micrOMEGAs [46] is used to calculate specific observables for each model-set in order to compare them with the experimental restrictions. The following constraints 
impact especially the neutralino and chargino mixing and can also influence their decay to the lightest Higgs boson.

- From the WMAP and the Planck data we adopt the cold dark-matter (DM) relic density in the universe [47, 48]. We select a region corresponding to the last Planck central value $0.1186 \pm 0.0031$ including an $10 \%$ (upper) and $20 \%$ (lower) theoretical uncertainty: ${ }^{3}$

$$
0.094<\Omega_{c} h^{2}<0.131
$$

- The limits from the 85.3 days Large Underground Xenon (LUX) data [49] are taken into account. To compare the calculated proton/neutron cross sections $\sigma^{(p / n)}$ to the experimental limits, we use a normalized cross section for a point-like nucleus [50]:

$$
\sigma=\frac{\left(Z \sqrt{\sigma^{(p)}}+(A-Z) \sqrt{\sigma^{(n)}}\right)^{2}}{A^{2}}
$$

with $A$ and $Z$ the mass number and atomic number of the target. In our case the target is xenon with $A=131$ and $Z=54$.

- We implement the LHCb and CMS measurements of the branching ratio of the strange $\mathrm{B}$ meson to two muons $[51,52]$ by demanding

$$
B R\left(B_{s}^{0} \rightarrow \mu^{+} \mu^{-}\right)=\left(3.0_{-0.9}^{+1.0}\right) \times 10^{-9} .
$$

- We impose the LEP limits on the invisible width of the $Z$ boson and on the SUSY particle masses as implemented in micrOmegas.

The WMAP/Planck results place severe constraints on the models as can be seen in figure 2. The LSP's of the surviving models turn out to be mostly Binolike, with a relatively low mass, and to a lesser extent Higgsinolike or Winolike, with a relatively high mass. This is caused by the possibility of coannihilation of the LSP together with the lightest chargino or next-to-lightest neutralino, which is mostly absent for Binolike LSP's. In order to reduce the efficiency of the coannihilation and have a DM relic density that is not too low, Higgsinolike and Winolike LSP's are substantially heavier than Binolike LSP's. The occurrence of Winolike LSP's is suppressed within the simulated parameter space, since in that case the coannihilation turns out to be very efficient. Among the useful models that survive the WMAP/Planck constraint we have found only a few with a Winolike LSP.

Having checked the impact of the WMAP/Planck constraint, we now impose the LUX limits on the surviving models. The additional impact of the LUX limits is much smaller, as can be seen in figure 3 where the LUX experimental limits are imposed. Given the WMAP/Planck and LUX constraints, the $B_{s}$ and LEP constraints have little additional impact on the number of viable models. Notable exceptions are the surviving models in figure 3 with a very light Higgsinolike LSP, which are removed by the LEP constraints on the lightest chargino mass.

\footnotetext{
${ }^{3} \mathrm{~A}$ value of approximately $10 \%$ is due to uncertainties entering the calculations of the relic density from SUSY parameters. The lower uncertainty is slightly larger, in order to include the possibility to have small additional sources of Dark Matter.
} 


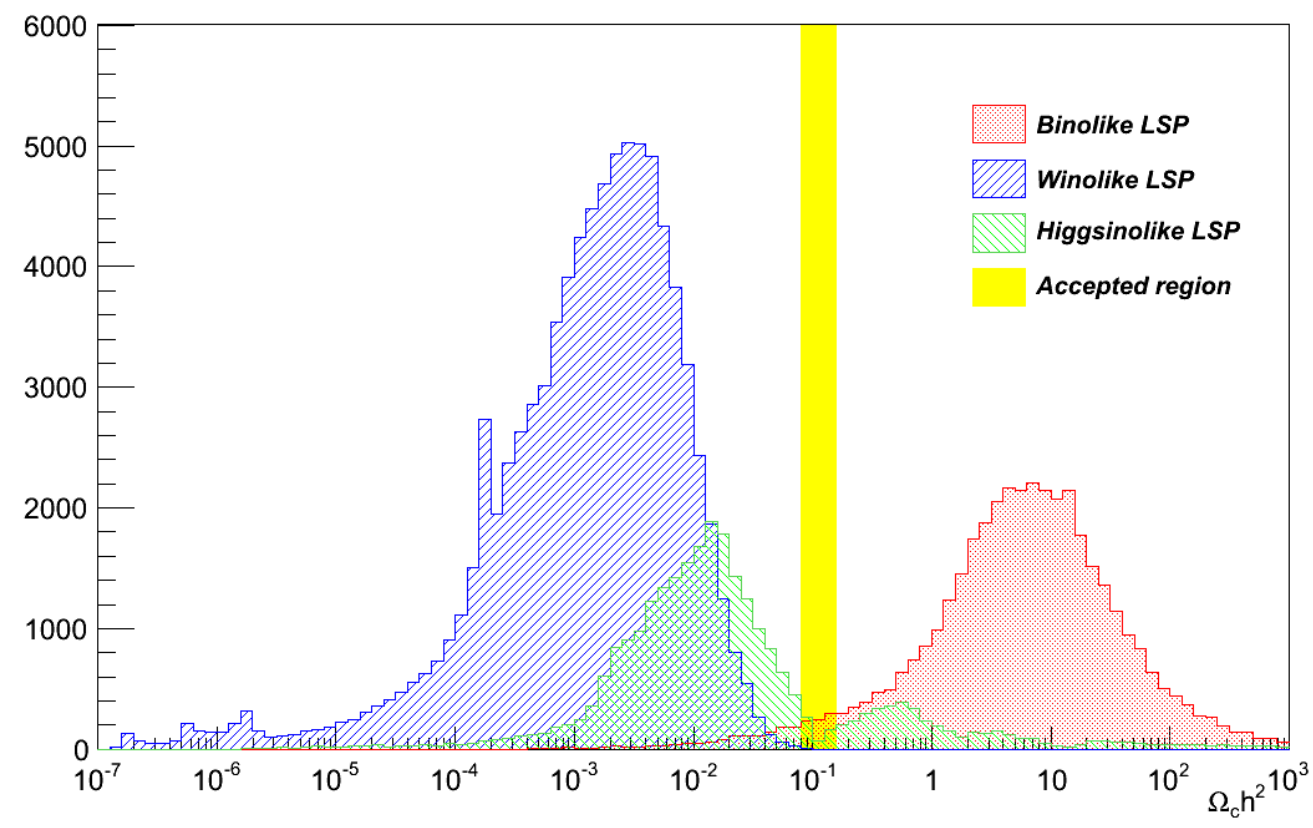

Figure 2. Dark Matter relic density $\Omega_{c} h^{2}$ obtained from the 19 parameter pMSSM models compared with the accepted region. The number of models is shown as a function of $\Omega_{c} h^{2}$.

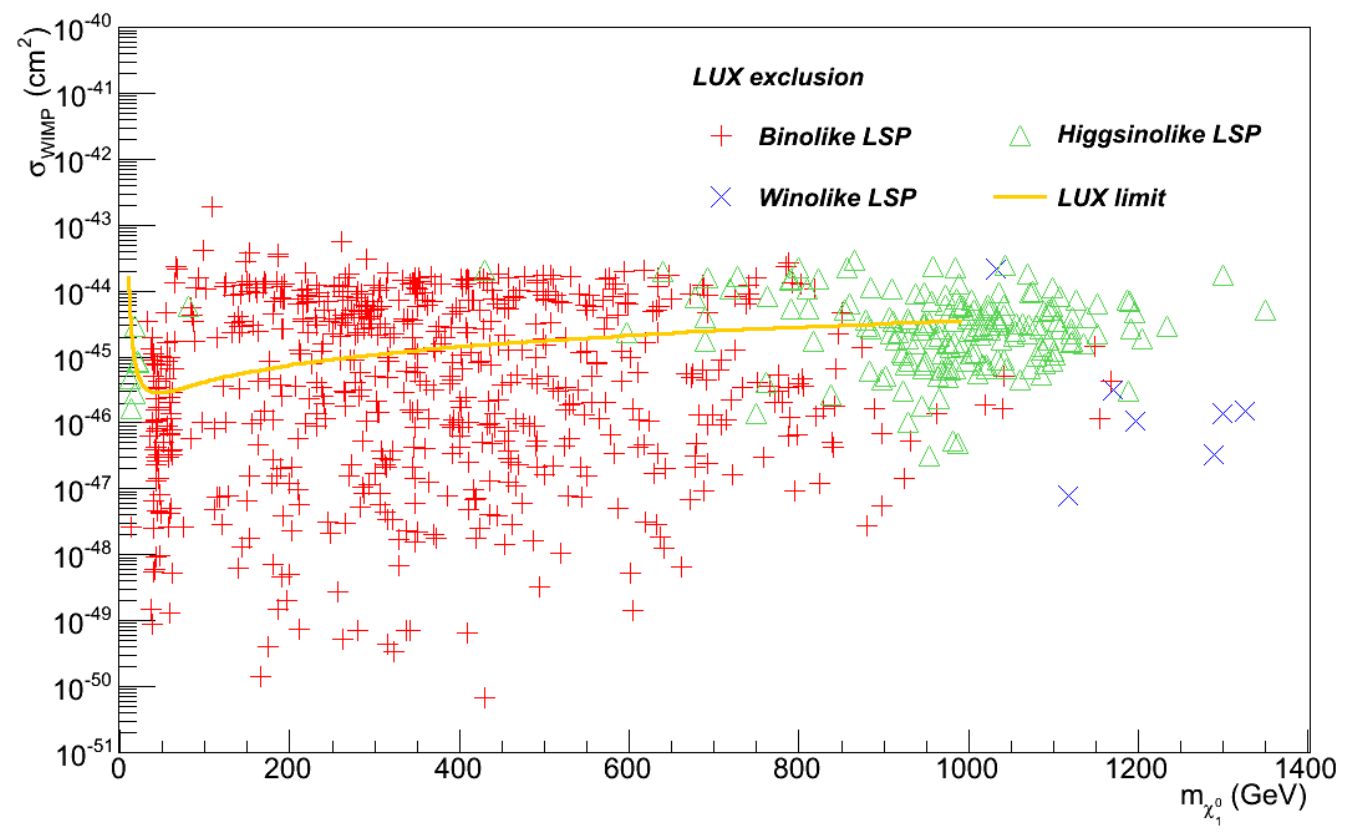

Figure 3. Upper limits on the LSP-nucleon scattering cross section for the LUX experiment, compared with the 19 parameter pMSSM after the WMAP/Planck constraint. LEP constraints are not yet applied. 
After a first iteration about 250 models survived. These models were again used to construct a posterior for a second particle-filter iteration, producing in total about 430 models that fulfil all constraints. As expected the success rate with this posterior is increased.

\subsection{ATLAS constraints: event generation, fast simulation and analysis}

The remaining model-sets are compared with recent constraints from LHC SUSY searches at $7 \mathrm{TeV}$ and $8 \mathrm{TeV}$ centre-of-mass energies. Most important are constraints from searches for squarks and/or gluinos and chargino-neutralino production. Searches for heavy Higgs production had no influence on the remaining models. The mass constraint on the $h^{0}$ boson demands either large $M_{A}$ or very heavy top squarks. In fact, the model with the lightest $A^{0}$ boson in our sample has $M_{A} \approx 330 \mathrm{GeV}$. Since $\tan \beta=6.9$ is relatively small for this model, it is not excluded by the ATLAS and CMS searches for heavy Higgs production [53, 54].

The limits of the ATLAS experiment on light squarks, gluinos and chargino-neutralino production are implemented by emulating the ATLAS analysis chain. Events from LHC collisions are generated for each pMSSM model and the detector response is simulated by a fast detector simulation. The acceptance and efficiency is determined by applying the most important ATLAS analysis cuts on the simulated events. Finally, these numbers are used to calculate the expected number of signal events for each signal region and analysis. Subsequently, these expected yields are compared to the model-independent 95\% C.L. limits provided by ATLAS.

PYTHIA 6.4 [55] is used for the event simulation of proton-proton $(p p)$ collisions at a $7 \mathrm{TeV}$ and $8 \mathrm{TeV}$ centre-of-mass energy. All SUSY production processes are enabled. For every model point and each centre-of-mass energy 10000 events are generated, which we found to be enough even for the models with the smallest selection efficiencies. To get as close as possible to the ATLAS analysis we use DELPHES 3.0 [56] as a fast detector simulation with the default ATLAS detector card, modified by setting the jet cone radius to 0.4. The PYTHIA output is read in by DELPHES in HepMC format, which is produced by HepMC 2.04.02 [57]. The object reconstruction is done by DELPHES, which uses the same anti- $\mathrm{k}_{T}$ jet algorithm [58] as ATLAS. Also included in the reconstruction are isolation criteria for electrons and muons. We do not emulate pile-up events.

The $7 \mathrm{TeV}$ analysis implementation is identical to ref. [59]. The selection efficiencies of our own implementation were compared to ATLAS in ref. [59] and were found to agree within uncertainties. For this study the implementation used in ref. [59] was updated using the recent $8 \mathrm{TeV}$ selection criteria. For the chargino-neutralino searches the SR0 $\tau$ a selection with all 20 bins was implemented as described in ref. [60]. For the squark and gluino searches all 13 signal regions without explicit $W$ selection of ref. [61] are considered. In order to check constraints from multi-b-jet searches we included also signal region SR$0 \ell$-A from [62].

Preliminary direct searches for decays into $h^{0}$ bosons from neutralinos do not influence the remaining models. The mass of the lightest neutralino with a sizeable branching ratio to Higgs bosons is about $185 \mathrm{GeV}$ and the mass of the LSP is at least $40 \mathrm{GeV}$. This is well beyond the exclusion reach of the ATLAS and CMS searches in these channels [34-36]. 


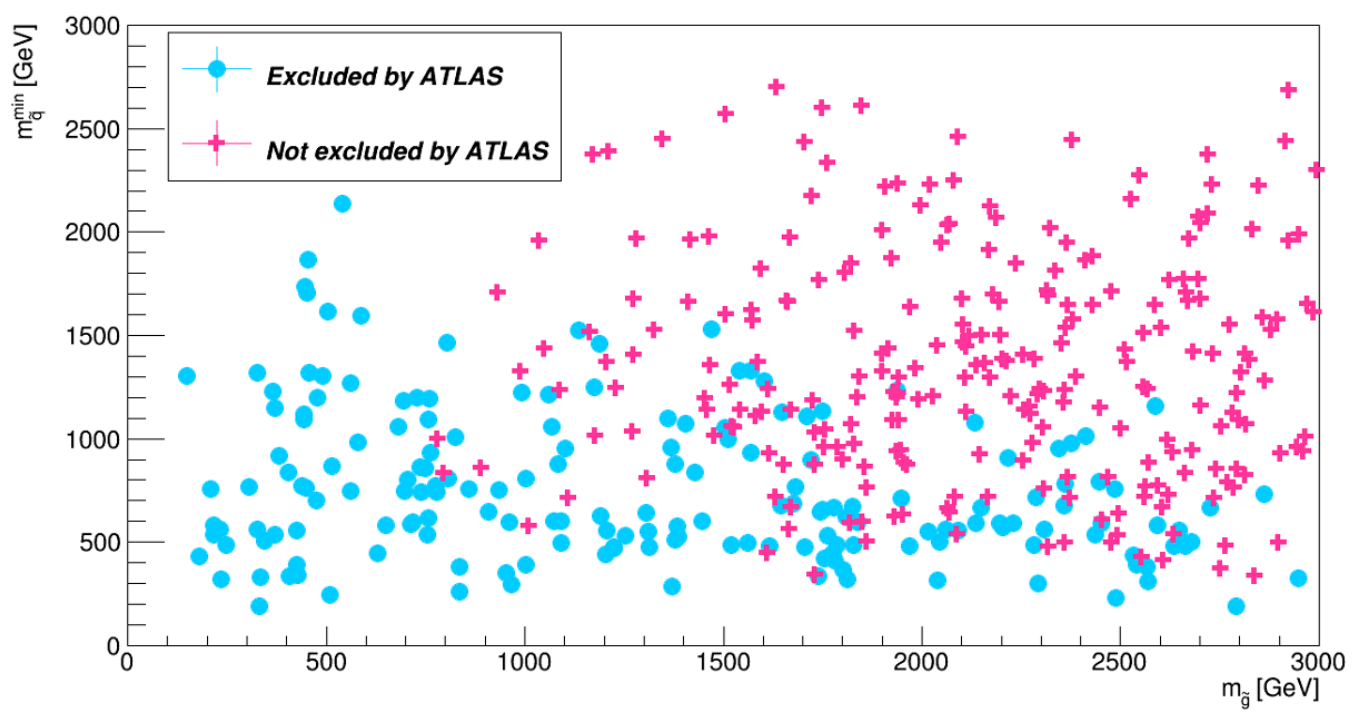

Figure 4. Lightest light-flavoured squark mass against the gluino mass for models that survive the ATLAS constraints (red crosses) and models that are excluded by ATLAS (blue dots).

After the event selection, the event counts are scaled to the luminosities considered in the analyses with leading order cross sections as provided by Pythia. The limits on the effective cross sections given by the ATLAS analyses are used to calculate a limit on the number of signal events passing the cuts. No attempt was made to include theoretical uncertainties. In the studied SUSY mass range these uncertainties are small compared to the differences of the ATLAS and DELPHES setups and would not change drastically any conclusion of this work.

In the end, 252 of the models passed all selection criteria. Figure 4 shows the excluded and non-excluded models as a function of the gluino mass and the minimal mass of the first and second generation squarks $m_{\tilde{q}}^{\min }$. Most excluded model points are due to limits on squarks and gluinos and have squark or gluino masses below about $1500 \mathrm{GeV}$, in agreement with current LHC limits. All models with a gluino mass below $750 \mathrm{GeV}$ are excluded. Remarkably, a large fraction of models with low squark masses is still allowed. One wellknown reason for this is that the lightest squark can be compressed with the $\widetilde{\chi}_{1}^{0}$ as shown in figure 5. This leads to very soft jets from squark decays. The squarks might only be visible via mono-jet signatures.

The enhancement of Higgs production in the studied models leads to a second interesting feature that causes the fraction of non-excluded models in this study to be larger than previously found in other scans (e.g. in [63]). In many non-excluded models the lightest squarks are compressed with a heavy neutralino/chargino. To illustrate this we indicate the minimal mass of all first and second generation squarks and the gluino by $A$. Figure 6 shows the smallest difference $\min (\Delta A)$ between $A$ and the masses of all neutralinos and charginos as a function of $A$ (given that the neutralino or chargino mass is smaller than $A$ ). In contrast to figure 5 , all non-excluded models with $A<800 \mathrm{GeV}$ have a mass difference $\Delta A$ below $300 \mathrm{GeV}$, which implies that many squarks are compressed with $\widetilde{\chi}_{2,3,4}^{0}$ or a chargino. 


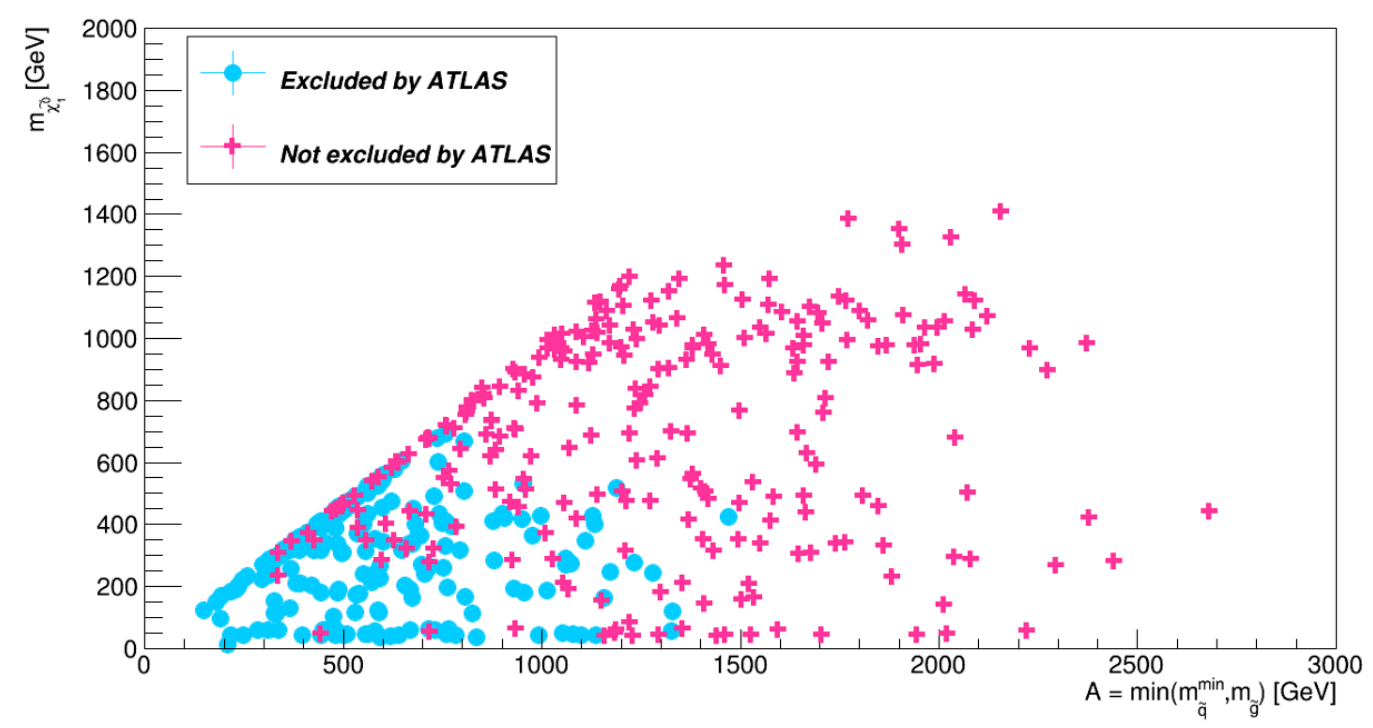

Figure 5. The excluded and non-excluded models as a function of the LSP mass and the minimum of the gluino mass and the masses of the first and second generation squarks $\min \left(m_{\tilde{q}}^{\min }, m_{\tilde{g}}\right)$.

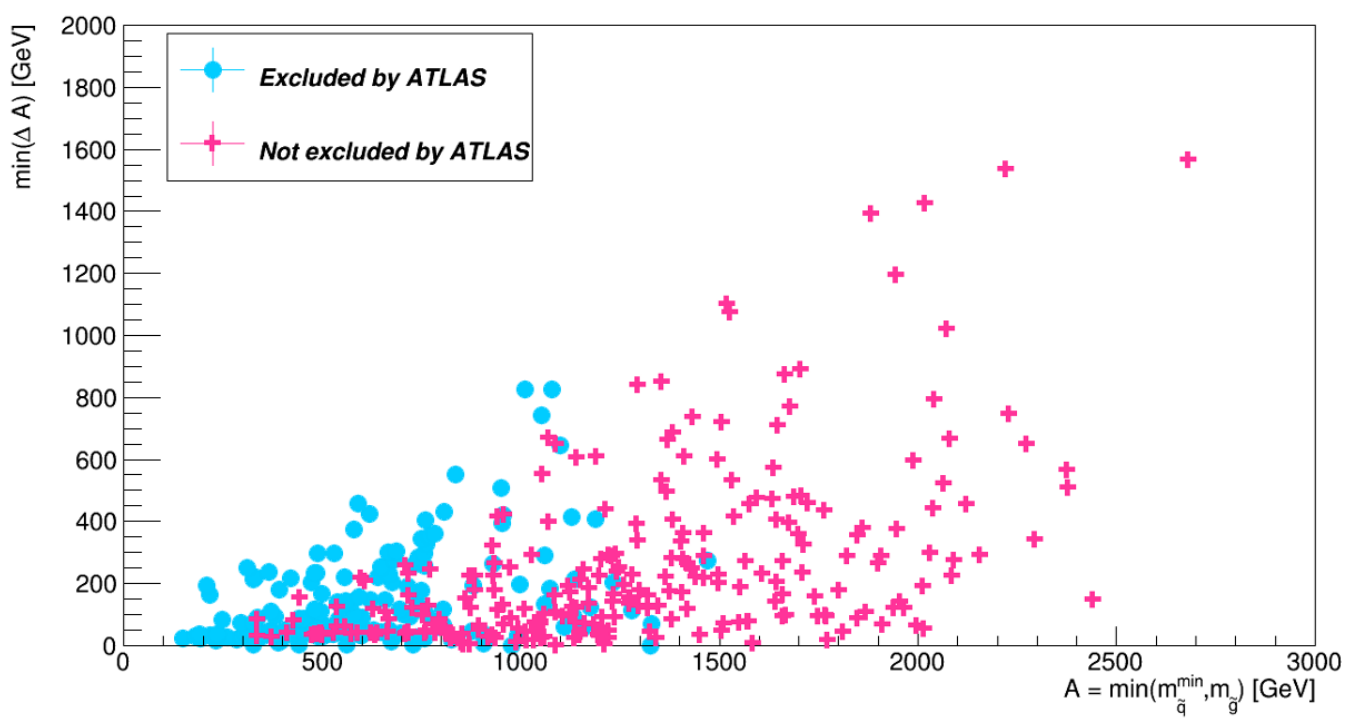

Figure 6. The same as in figure 5, but this time the LSP mass is replaced by the smallest difference $\min (\Delta A)$ between $A=\min \left(m_{\tilde{q}}^{\min }, m_{\tilde{g}}\right)$ and the masses of all neutralinos and charginos (given that the neutralino or chargino mass is smaller than $A$ ).

As discussed in section 2, in many cases the squarks do not directly decay to the LSP, especially in the model points selected for this study. If e.g. the LSP is Binolike, the lightest $\widetilde{q}_{L}$ prefers to decay (if kinematically allowed) into the heavier Winolike neutralino or chargino. This is caused by a $3 c_{w} / s_{w} \approx 5.5$ enhancement factor of the weak coupling with respect to the hypercharge coupling. If the squark happens to be compressed with the 
Winolike neutralino, jets from the squark decays are also soft and the remaining signature is determined by the branching ratios of the heavier Winolike neutralino. In these models the Winolike neutralino can have a large branching ratio to Higgs bosons. If it is a $\widetilde{\chi}_{4}^{0}$, then decays to charginos are also possible. If the chargino decay is dominant, SUSY searches with leptons might be sensitive to these points. Searches asking for one lepton in the final state typically exclude simplified models with degenerate squarks decaying to charginos if $m_{\widetilde{q}}<800 \mathrm{GeV}$ and $m_{\widetilde{\chi}_{1}^{0}}<300 \mathrm{GeV}$ (see e.g. [64]). After applying all other search constraints we find no model that fulfills these requirements. If the Winolike neutralino is a $\widetilde{\chi}_{2}^{0}$, then explicit searches for Higgs production from squarks might give a unique discovery possibility. Similar multi-step decays are possible in other cases as outlined in section 2.

\section{Analysis of the candidate models}

\subsection{Branching ratios}

The branching ratios of all SUSY particles for decays into the lightest Higgs boson $h^{0}$ have been determined for all surviving models. These branching ratios include also multi-step decays to the Higgs boson, i.e. particles can have a non-zero branching ratio even if they do not couple to the Higgs boson directly. We show in figure 7 the branching ratio for all MSSM particles to the light Higgs boson $h^{0}$. All models are shown in grey in order to indicate the ranges of these branching ratios. The possible decay processes have been described in more detail in section 2.

The sfermions can have decay branching ratios of up to 0.4, with the values for left- and right-handed sfermions strongly varying from model to model. The $\widetilde{b}_{2}$ and $\widetilde{t}_{2}$ squarks have a larger branching ratio due to the direct decay $\widetilde{f}_{2} \rightarrow \widetilde{f}_{1}+h^{0}$. As explained in section 2 , the $\widetilde{\chi}_{2,3}^{0}$ neutralinos can have branching ratios close to unity. The $\widetilde{\chi}_{2}^{ \pm}$charginos can have a branching ratio that substantially exceeds 0.35 due to multi-step decays. The branching ratios of the heavy Higgs bosons range up to $\sim 0.4$ due to direct as well as multi-step decays. Some models with interesting features are shown in colour. These models, which are labeled $\mathrm{A}-\mathrm{E}$, are shown in table 3 and are discussed in more detail below.

If we go one step further and take a look at all supersymmetric particles that decay into at least two Higgs bosons, the heaviest neutralino has the highest branching ratio, as can be seen in figure 8. Although it is not the preferred decay channel, it can decay into a $\widetilde{\chi}_{2,3}^{0}$, which can subsequently decay into a LSP. Both decays can produce one $h^{0}$ boson. The sfermions can decay into two $h^{0}$ bosons via the intermediate decay into a heavy neutralino. The $H^{0}$ boson can directly decay to two $h^{0}$ bosons, the $A^{0}$ and $H^{ \pm}$bosons decay via heavy neutralinos/charginos. The $\widetilde{t}_{2}$ top squark can decay to $\widetilde{t}_{1}+h^{0}$ and $\widetilde{t}_{1}$ can subsequently decay to one more $h^{0}$.

\subsection{Event generation, fast simulation and analysis}

In order to determine the phenomenological relevance of $h^{0}$ production via SUSY processes, the LHC production rate needs to be determined. The generation of simulated events of $p p$ collisions at a centre-of-mass energy of $14 \mathrm{TeV}$ for each candidate model utilises 


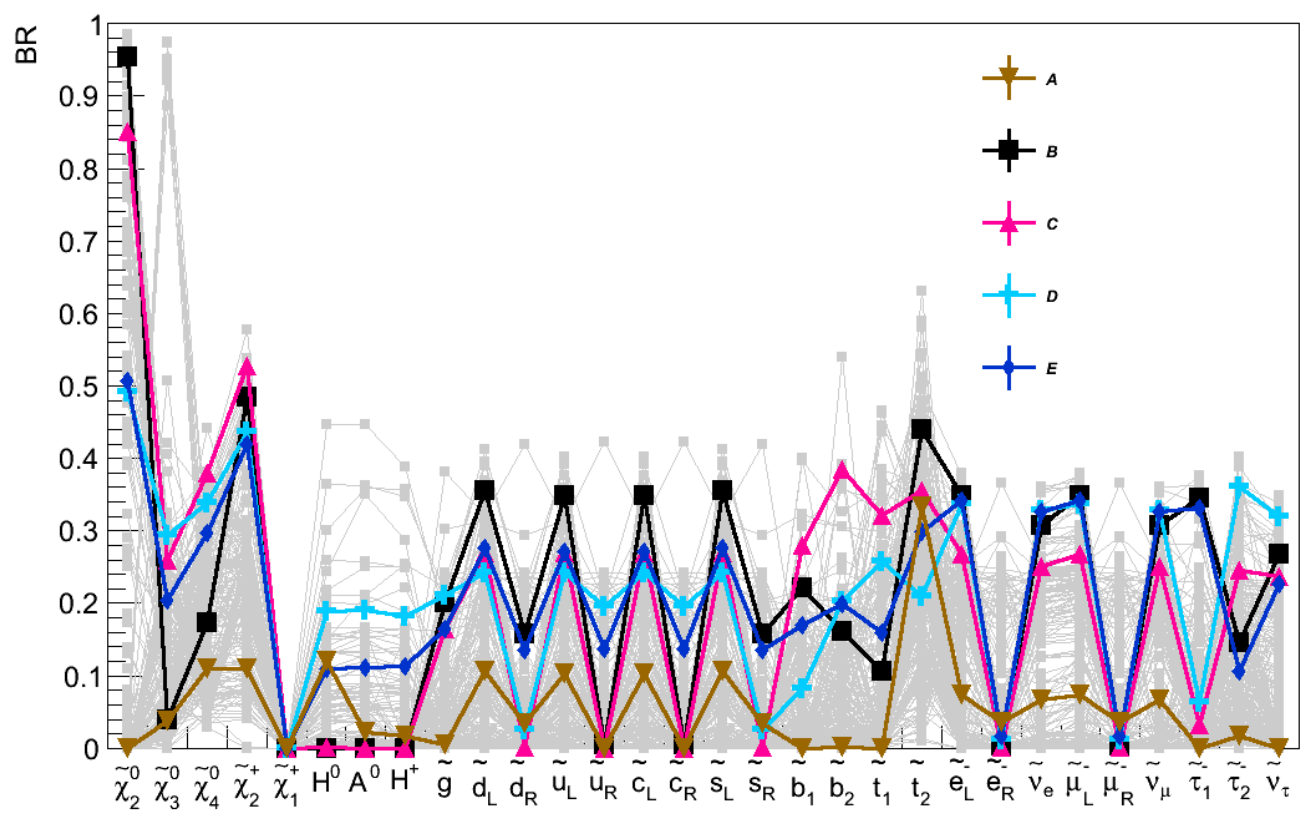

Figure 7. Branching ratio of all supersymmetric particles for decays into at least one $h^{0}$ boson for the candidate models that survive all constraints.

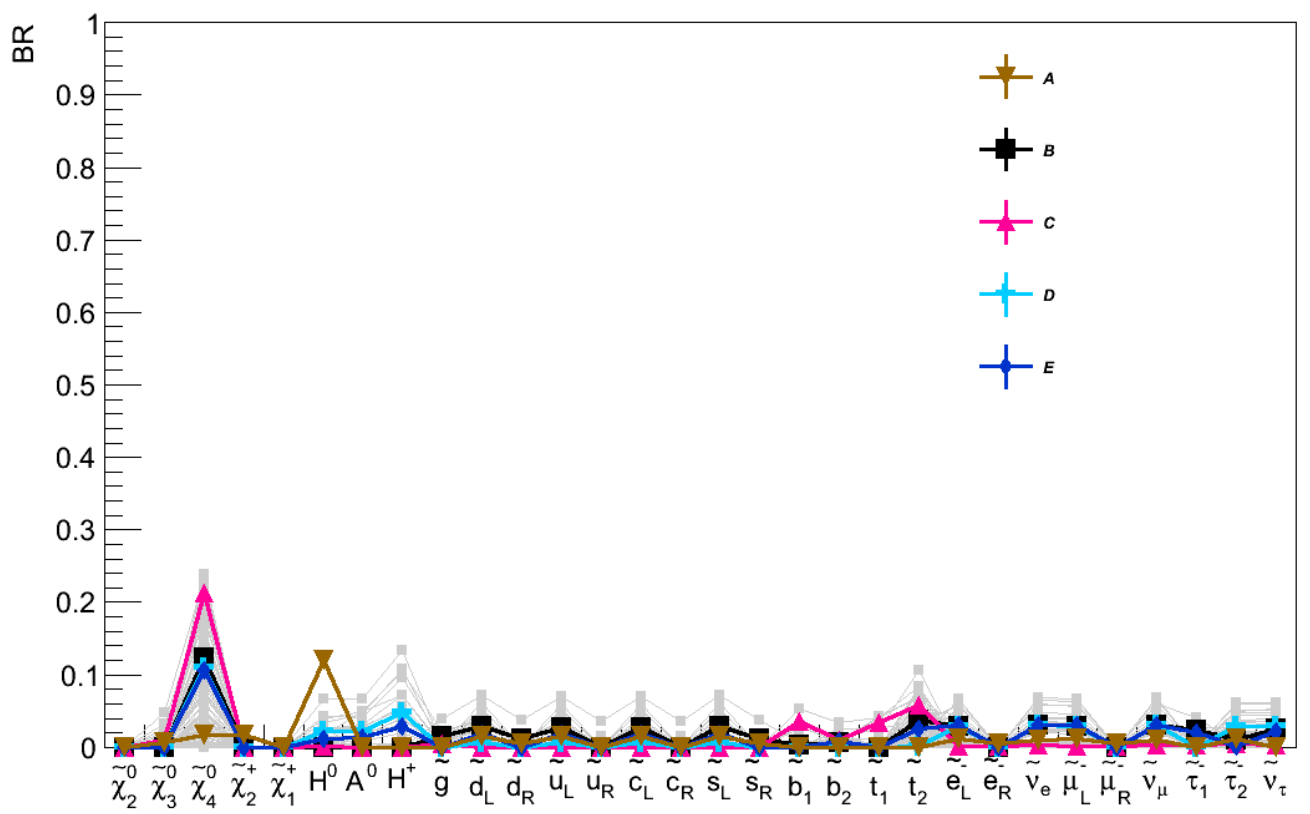

Figure 8. Branching ratio of all supersymmetric particles for decays into at least two $h^{0}$ bosons for the candidate models that survive all constraints. 
PYTHIA6.4, HepMC 2.04.02 and DELPHES 3.0, just as described in section 3.4. In the simulation the branching ratio of the $h^{0}$ boson into two photons has been set to unity manually. This is done in order to prevent interference of jets or leptons originating from $h^{0}$ decays when analysing the jet multiplicities in the final states later on in this section. All other decay channels of the $h^{0}$ boson have been assigned a zero branching ratio. This does not affect the total $h^{0}$ production rates. A total number of 100000 events are generated for each of the approximately 250 candidate models that survive all previous constraints. All SUSY production processes are enabled.

\subsection{Determining the expected number of events with Higgs bosons}

The number of expected Higgs $\left(h^{0}\right)$, di-Higgs and tri-Higgs events is calculated for an integrated luminosity of $10 \mathrm{fb}^{-1}$ for each SUSY production process. For each model point the branching ratios to the $h^{0}$ as well as the SUSY production cross sections $\sigma_{I S U B_{i}}$ for each subprocess $I S U B_{i}$ are considered. All cross sections are determined by PYTHIA. No attempt was made to include NLO corrections. In general these NLO corrections would further increase the production rate. So, in that sense our estimates are conservative. The number of events with at least one, two or three $h^{0}$ bosons is calculated as

$$
\begin{aligned}
N_{\text {events }}^{\geq 1 h^{0}}= & \mathcal{L}_{\text {int }} \cdot \sigma_{I S U B_{i}} \cdot\left[B R_{\widetilde{C}}\left(\widetilde{C} \rightarrow \geq 1 h^{0}+X\right)\right. \\
& \left.+B R_{\widetilde{C}}\left(\widetilde{C} \rightarrow 0 h^{0}+X\right) \cdot B R_{\widetilde{D}}\left(\widetilde{D} \rightarrow \geq 1 h^{0}+Y\right)\right], \\
N_{\text {events }}^{\geq 2 h^{0}}= & \mathcal{L}_{\text {int }} \cdot \sigma_{I S U B_{i}} \cdot\left[B R_{\widetilde{C}}\left(\widetilde{C} \rightarrow \geq 1 h^{0}+X\right) \cdot B R_{\widetilde{D}}\left(\widetilde{D} \rightarrow \geq 1 h^{0}+Y\right)\right. \\
& +B R_{\widetilde{C}}\left(\widetilde{C} \rightarrow 0 h^{0}+X\right) \cdot B R_{\widetilde{D}}\left(\widetilde{D} \rightarrow \geq 2 h^{0}+Y\right) \\
& \left.+B R_{\widetilde{C}}\left(\widetilde{C} \rightarrow \geq 2 h^{0}+X\right) \cdot B R_{\widetilde{D}}\left(\widetilde{D} \rightarrow 0 h^{0}+Y\right)\right], \\
N_{\text {events }}^{\geq 3 h^{0}}= & \mathcal{L}_{\text {int }} \cdot \sigma_{I S U B_{i}} \cdot\left[B R_{\widetilde{C}}\left(\widetilde{C} \rightarrow \geq 1 h^{0}+X\right) \cdot B R_{\widetilde{D}}\left(\widetilde{D} \rightarrow \geq 2 h^{0}+Y\right)\right. \\
& +B R_{\widetilde{C}}\left(\widetilde{C} \rightarrow \geq 2 h^{0}+X\right) \cdot B R_{\widetilde{D}}\left(\widetilde{D} \rightarrow 1 h^{0}+Y\right) \\
& +B R_{\widetilde{C}}\left(\widetilde{C} \rightarrow 0 h^{0}+X\right) \cdot B R_{\widetilde{D}}\left(\widetilde{D} \rightarrow \geq 3 h^{0}+Y\right) \\
& \left.+B R_{\widetilde{C}}\left(\widetilde{C} \rightarrow \geq 3 h^{0}+X\right) \cdot B R_{\widetilde{D}}\left(\widetilde{D} \rightarrow 0 h^{0}+Y\right)\right] .
\end{aligned}
$$

\subsubsection{Higgs production via SUSY processes}

Figure 9 shows the rate of events with $\geq 1 h^{0}$ for all SUSY production processes normalized to an integrated luminosity of $10 \mathrm{fb}^{-1}$. The most important classes of production processes are squark-(anti)squark production, in particular for left-handed squarks (see figure 23 in the appendix), chargino-neutralino production (see also figure 19 in the appendix) and neutralino pair production (see also figure 20 in the appendix). Next in line are the associated production of a neutralino/chargino and a light squark (see also figures 21 and 22 in the appendix), and the production of pairs of bottom or top squarks (see also figure 24 in the appendix).

Due to the nature of the mixing matrix, $h^{0}$ production via neutralino-pair and charginoneutralino processes are correlated. Large Higgs production rates are possible if the heavier neutralinos/chargino are relatively light and decay to $h^{0}$. Examples are model $D$ and $E$ shown in table 3 . 


\begin{tabular}{|c|c|c|c|c|c|}
\hline 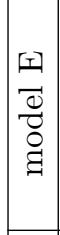 & 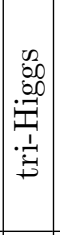 & 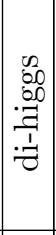 & 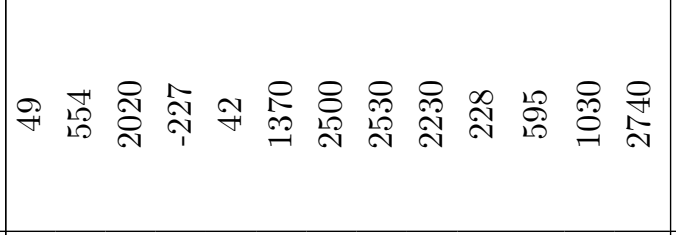 & 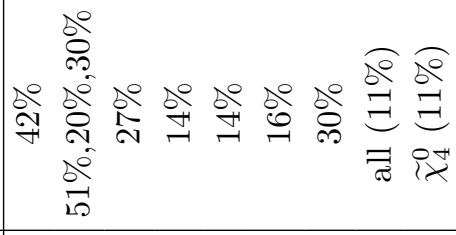 & 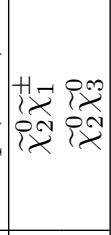 \\
\hline 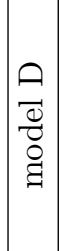 & 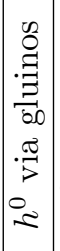 & 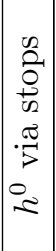 & 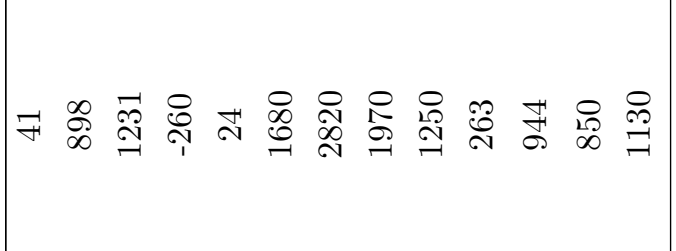 & 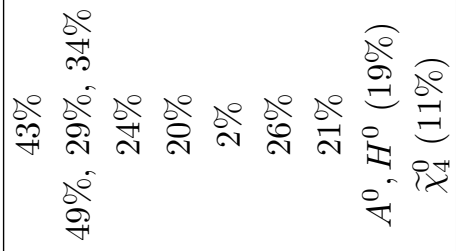 & 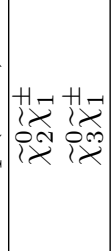 \\
\hline 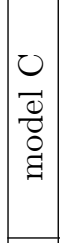 & 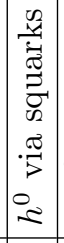 & 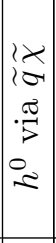 & 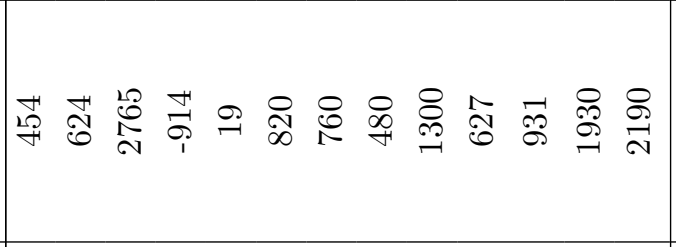 & 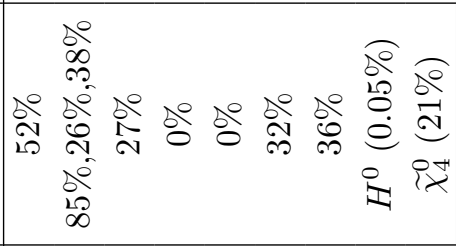 & 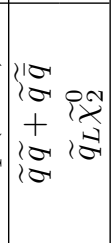 \\
\hline $\begin{array}{l}m \\
\vec{d} \\
0 \\
\vdots \\
0\end{array}$ & 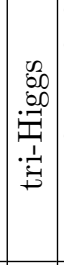 & 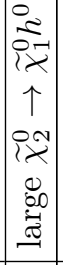 & 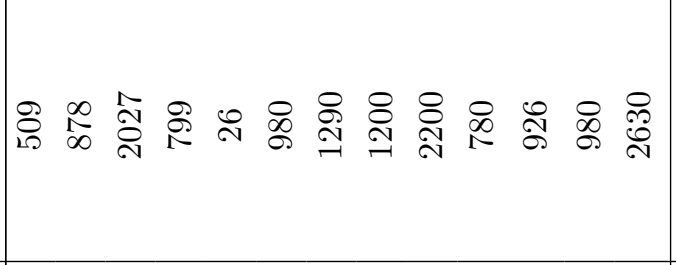 & 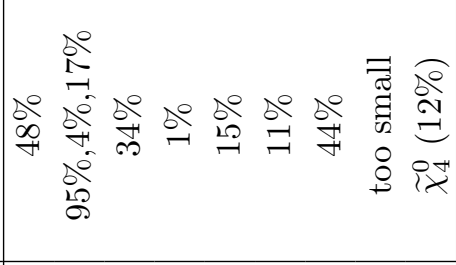 & 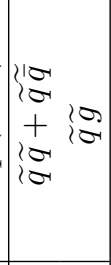 \\
\hline 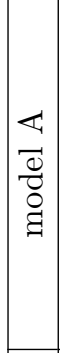 & 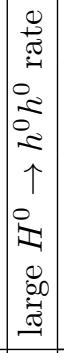 & 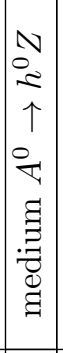 & 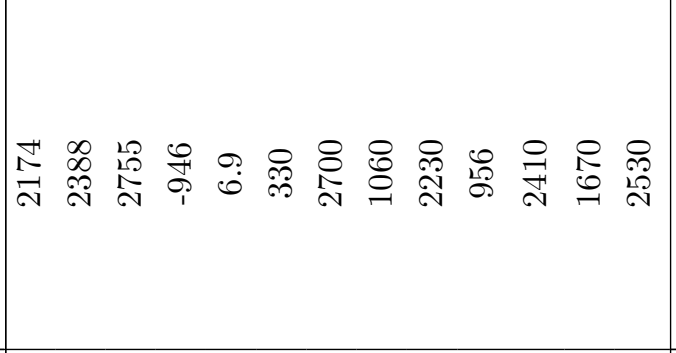 & 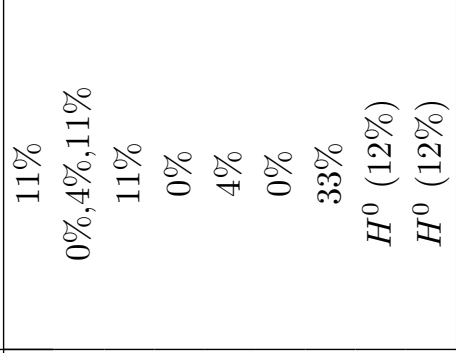 & i \\
\hline 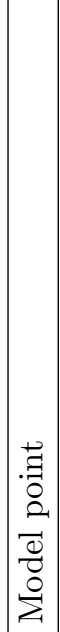 & 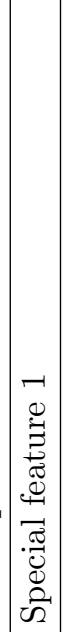 & 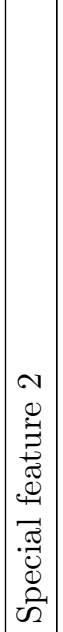 & 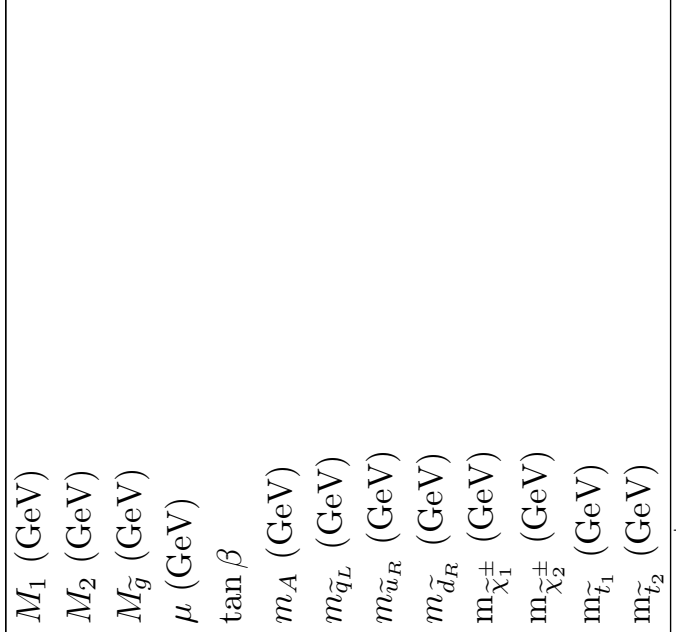 & 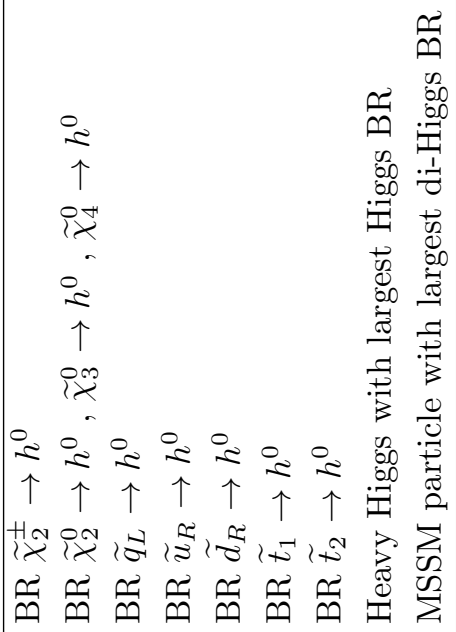 & 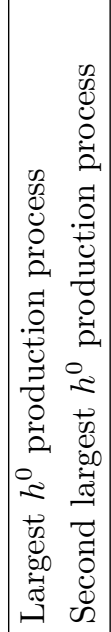 \\
\hline
\end{tabular}




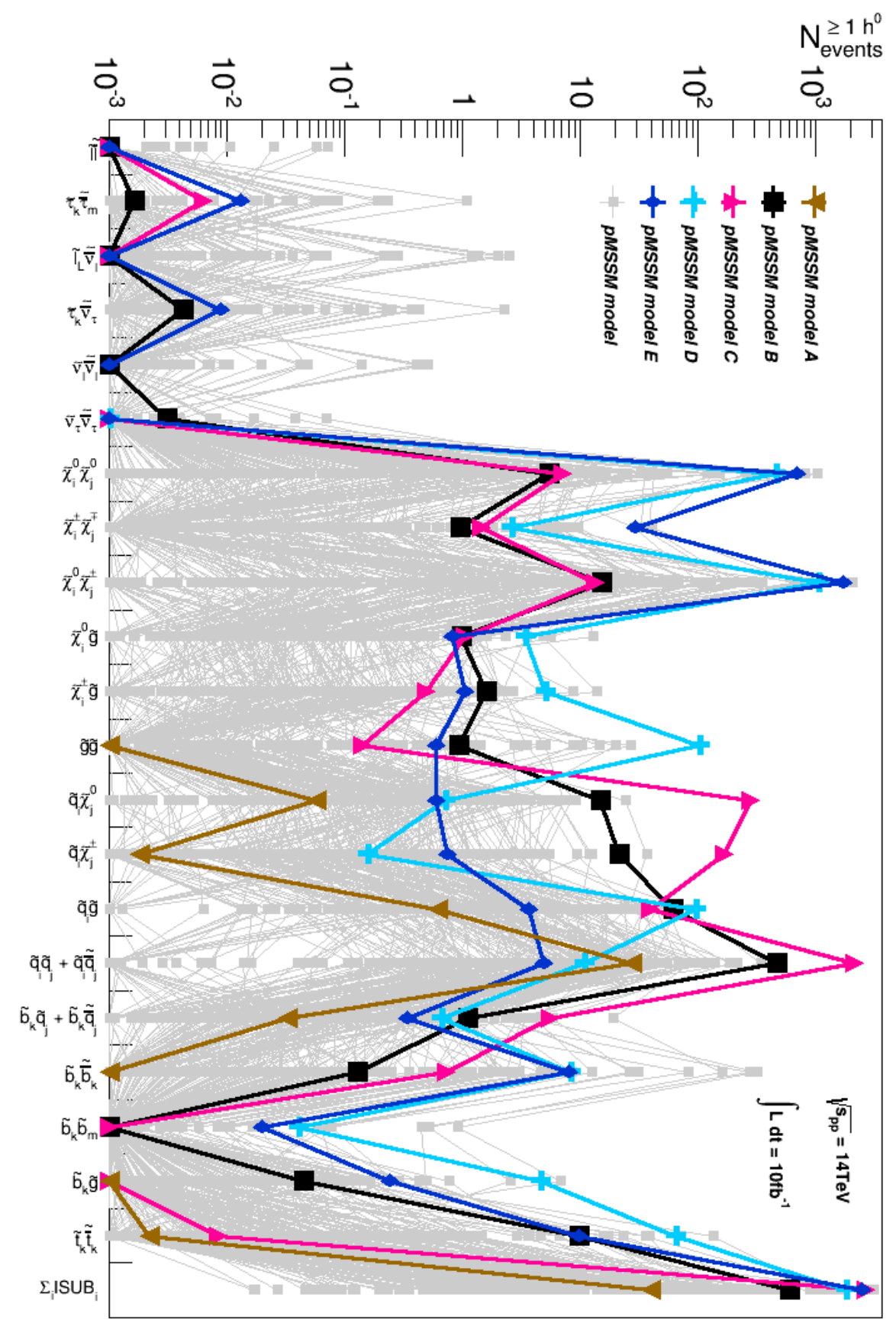

Figure 9. Number of expected events containing at least one $h^{0}$ boson in cascades of supersymmetric origin for all candidate models. The vertical axis indicates the supersymmetric final states of the primary interaction process. 
As can be seen in model $B$ or $C$, Higgs production from squarks can still be large when at the same time Higgs production via chargino/neutralino processes is supressed. This happens when the charginos and non-LSP neutralinos are too heavy (about $600-800 \mathrm{GeV}$ in model $B$ and $C$ ) for direct production. These heavy neutralinos/charginos can, however, still be produced in the decay of a slightly heavier squark. As described in sections 2 and 3 such squark decays might be dominant. Searching for squark production might then be the only possibility to detect these models. Similar neutralino/chargino decays are important to produce light Higgs bosons in bottom squark decays.

The associated production of a chargino/neutralino and a squark can also be interesting for $h^{0}$ production. For the same mass of the produced particles the associated production cross section is in between the (electroweak) chargino/neutralino production and the (strong) squark production. This process can be important if the mass of the chargino/neutralino is similar to the mass of the squark, i.e. if one of the squarks is rather light. As explained before squarks can still be light in our models, e.g. if the squark decays via a heavy chargino/neutralino rather than directly to $\widetilde{\chi}_{1}^{0}$. It is then difficult to detect the squarks in the conventional way at the LHC. An example is model $C$ where the lefthanded squark (mass $760 \mathrm{GeV}$ ) decays with $65 \%$ branching ratio into the $\widetilde{\chi}_{1}^{ \pm}$and with $30 \%$ branching ratio into $\widetilde{\chi}_{2}^{0}$ (both with a mass of $627 \mathrm{GeV}$ ). The $\widetilde{\chi}_{2}^{0}$ decays with $85 \%$ branching ratio into a $h^{0}$ boson.

Higgs-boson production via top squarks can be enhanced for light stops. An example is model $D$, which has a $\widetilde{t}_{1}$ mass of $850 \mathrm{GeV}$ and a $\widetilde{t}_{2}$ mass of $1130 \mathrm{GeV}$. Both stops decay to $h^{0}$ bosons predominantly via heavy neutralinos with branching ratios of $20-25 \%$. This gives rise to special final-state topologies, involving top quarks, (possibly multiple) Higgs bosons and missing transverse momentum. Higgs-boson production via gluinos proceeds through the decay into light squarks. In the case of model $D$, these light squarks are top squarks, leading to spectacular event topologies where the gluino (or even both gluinos) can decay into $h^{0} t \bar{t} \widetilde{\chi}_{1}^{0}$.

The most important Higgs production processes are summarized in table 4. In some models (e.g. model $D) h^{0}$ production is significant for almost all important SUSY production processes. When all contributions to $h^{0}$ production from SUSY interactions are summed up, realistic models are found that lead to about 3000 events with at least one $h^{0}$ for $10 \mathrm{fb}^{-1}$ of data. In almost all models a significant amount of missing tranverse momentum due to the LSP's is expected. This makes the events different from $h^{0}$ production via SM processes.

\subsubsection{Di-Higgs production via SUSY processes}

Since SUSY particles are pair produced and both particles can decay to a light Higgs boson, SUSY processes can be a significant source of events containing two $h^{0}$ bosons. We will show that this di-Higgs production rate can be significant. 


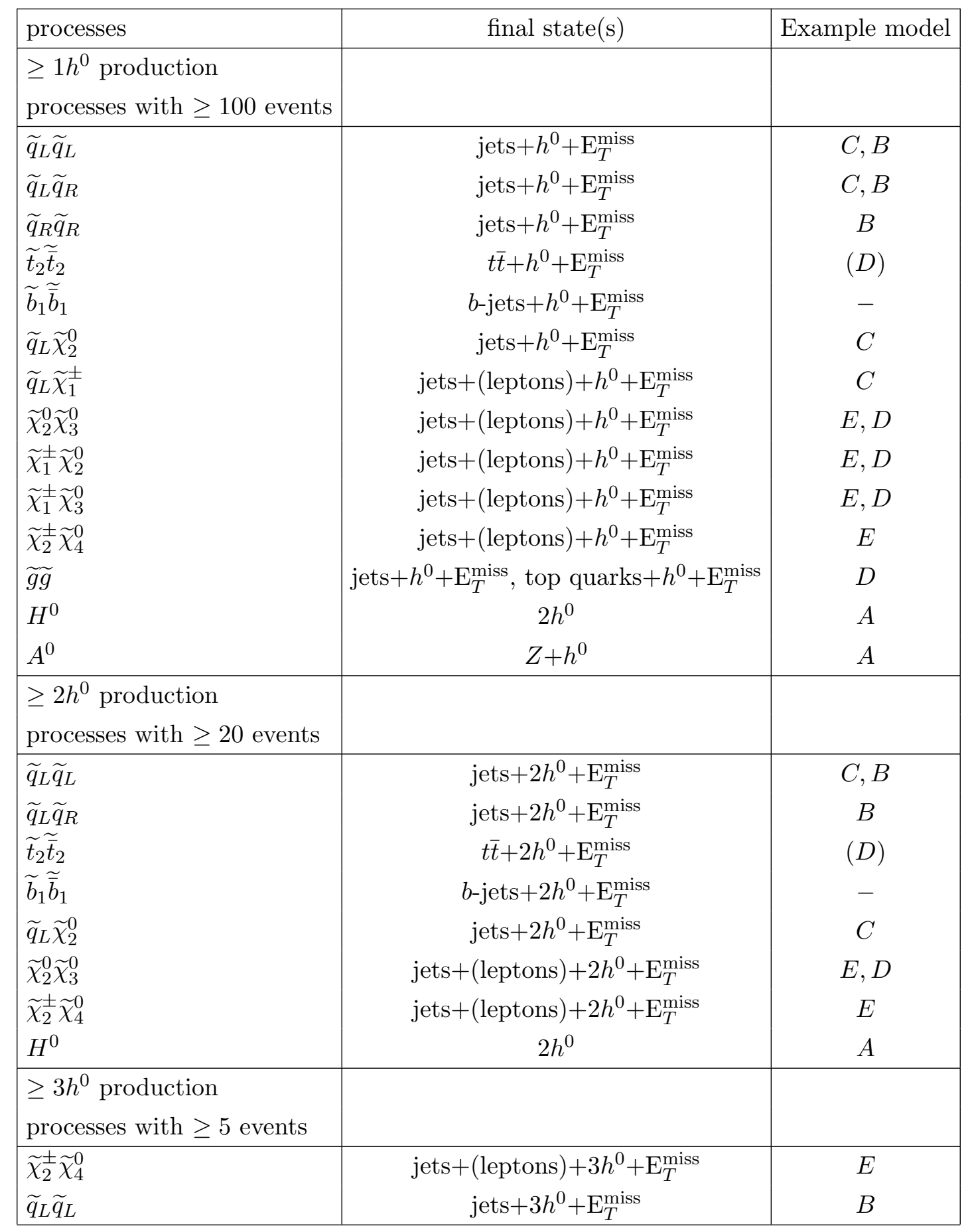

Table 4. List of most relevant SUSY processes to produce light Higgs bosons and the corresponding final-state topologies of interest, where $\mathrm{E}_{\mathrm{T}}^{\mathrm{miss}}$ indicates the presence of missing transverse momentum in the final state. Numbers are given for $10 \mathrm{fb}^{-1}$. 
Di-Higgs production is of utmost importance in the SM to measure the triple-Higgs coupling. As such, the measurement of di-Higgs production is a central research objective for the high luminosity phase of the LHC. The SM di-Higgs production has an expected next-to-next-to-leading order (NNLO) cross section of roughly $40 \mathrm{fb}$ [65], leading to about 400 events for $10 \mathrm{fb}^{-1}$. These events are very difficult to detect due to the enormous SM background rate.

In the MSSM another important source of di-Higgs events is the production of heavy Higgs bosons (see figure 25 in the appendix). Model $A$ predicts an enourmous rate of $>$ 2000 di-Higgs events, visible as a di-Higgs resonance. Heavy Higgs production is discussed separately in the next subsection. Di-Higgs events from processes involving SUSY particles are different due to the presence of large missing transverse momentum. The background from SM processes can be reduced by a large factor with cuts on this quantity. Figure 10 shows the di-Higgs production rate per SUSY process normalized to $10 \mathrm{fb}^{-1}$. Model $C$ predicts the largest SUSY production rate for di-Higgs events with about 350 events for $10 \mathrm{fb}^{-1}$. This rate can also be compared with 10 and 4.2 events expected from the SM $t \bar{t} h^{0} h^{0}$ or $Z h^{0} h^{0}$ processes, which have a cross section of $1.0 \mathrm{fb}$ at leading order $[66,67]$ and $0.42 \mathrm{fb}$ at NNLO [66], respectively. SUSY processes can therefore significantly enhance di-Higgs signatures in SM di-Higgs searches. Any deviation from the SM expectations in these searches needs therefore to be interpreted carefully, since deviations could be the result of SUSY decays.

The SUSY di-Higgs production is dominated by squark processes, followed by the direct production of heavy neutralinos/charginos. The most important SUSY di-Higgs production processes are summarized in table 4 .

\subsubsection{Tri-Higgs production via SUSY processes}

Due to the multi-step decays of heavy neutralinos there is the possibility that one heavy neutralino can decay to two $h^{0}$ bosons. The corresponding branching fractions were discussed in section 4.1 and $\widetilde{\chi}_{4}^{0}$ was found to be the dominant source. This makes it possible to produce three Higgs bosons in one event. Figure 11 shows the number of tri-Higgs events per SUSY process normalized to $10 \mathrm{fb}^{-1}$. Up to 20 tri-Higgs events can be produced, predominantly via squark production. The dominant tri-Higgs production processes are summarized in table 4. The SM tri-Higgs production cross section is $0.044 \mathrm{fb}$ [68] leading to an expectation of only 0.4 events for $10 \mathrm{fb}^{-1}$. Tri-Higgs production might become important for large luminosities or, after a LHC discovery, for determining e.g. the neutralino mixing matrix.

\subsection{The lightest Higgs boson from heavy-Higgs production processes}

For the sake of completeness, simulated events with primary interaction processes involving heavy Higgs particles are also investigated briefly. This investigation utilises the calculation of events with at least one $h^{0}$ boson according to equation (4.1), but this time only the branching ratios of the heavy Higgses into one or more light Higgs boson(s) are taken into account. 


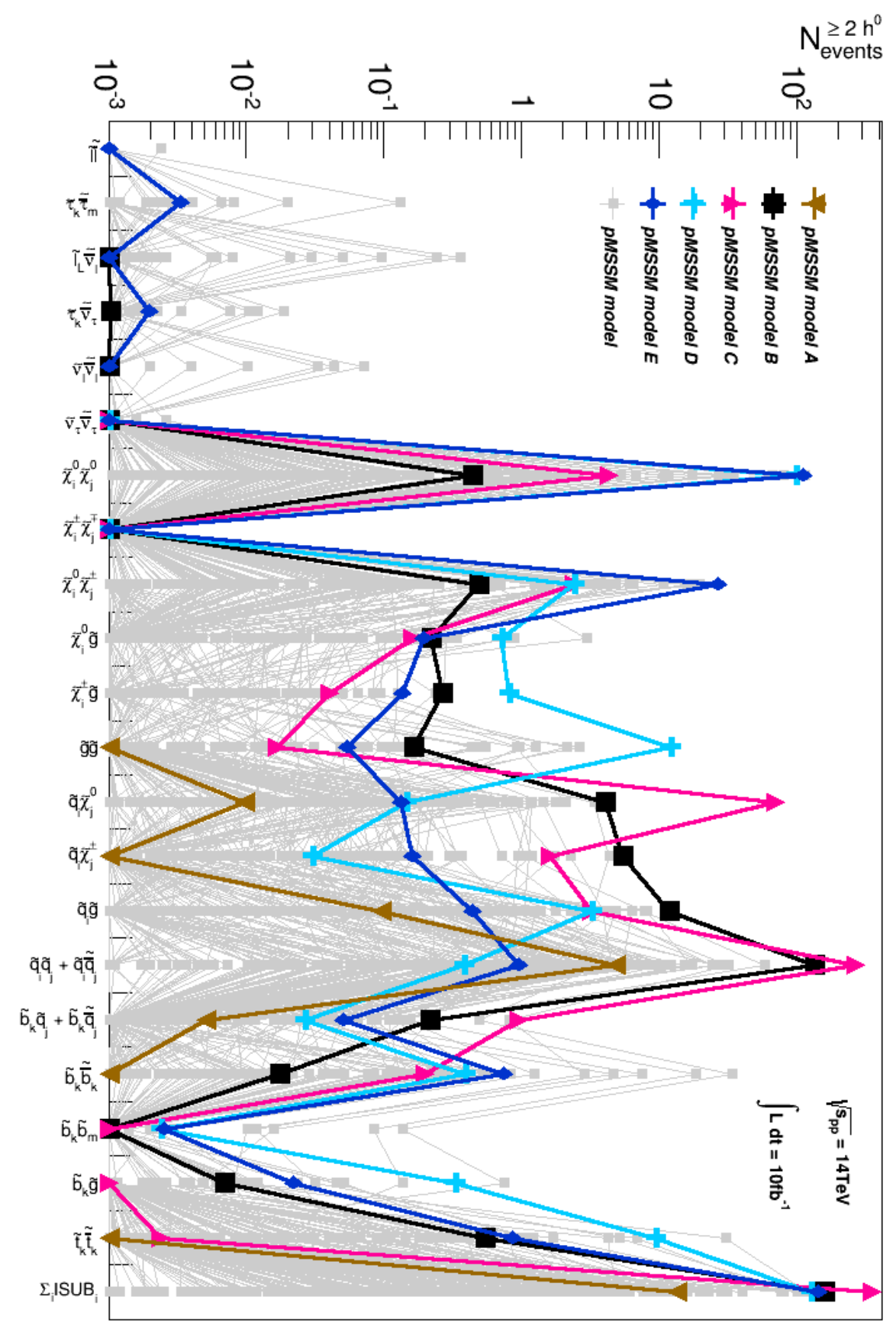

Figure 10. Number of expected events containing at least two $h^{0}$ bosons in cascades of supersymmetric origin for all candidate models. The vertical axis indicates the supersymmetric final states of the primary interaction process. 


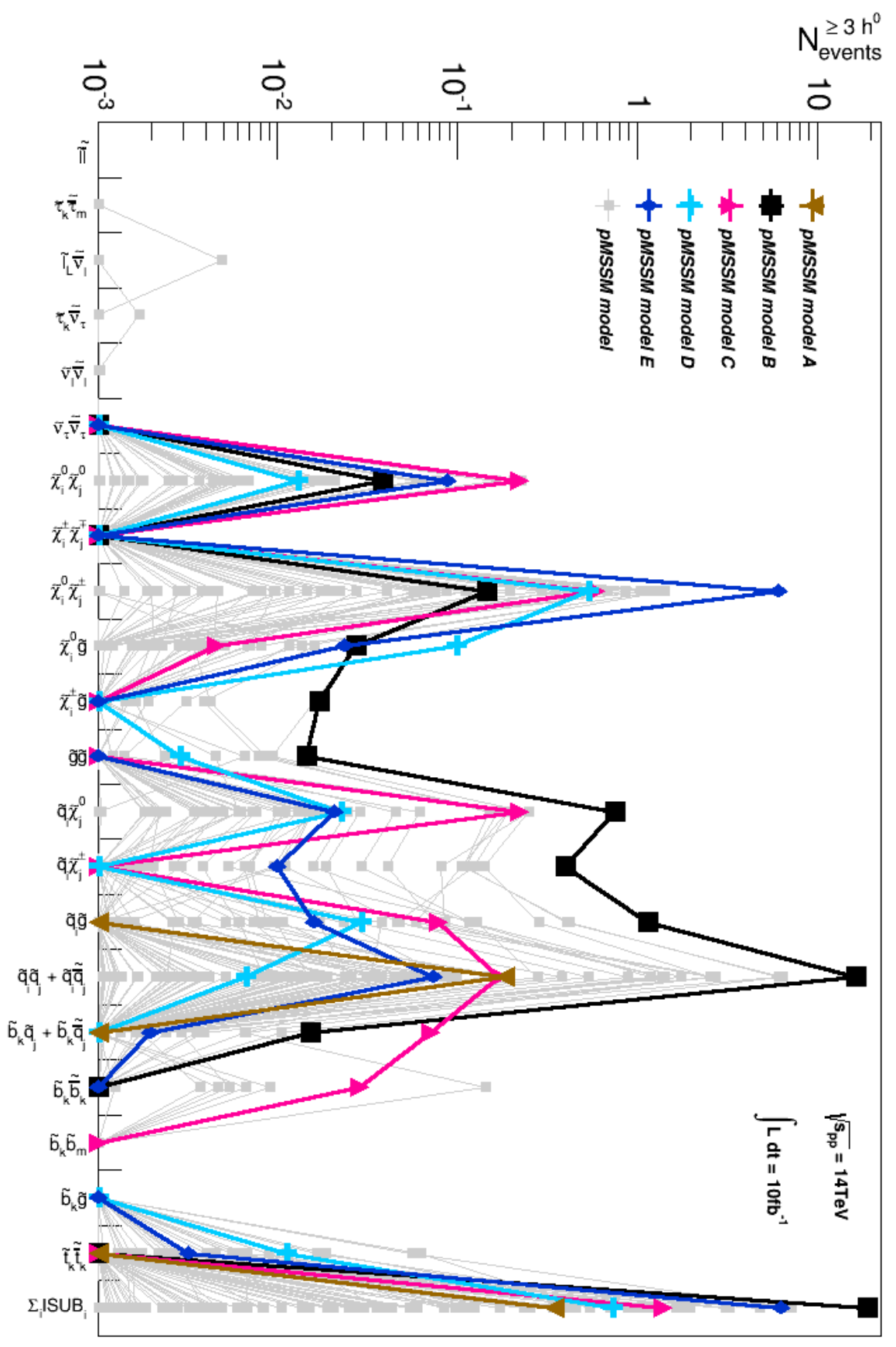

Figure 11. Number of expected events containing at least three $h^{0}$ bosons in cascades of supersymmetric origin for all candidate models. The vertical axis indicates the supersymmetric final states of the primary interaction process. 


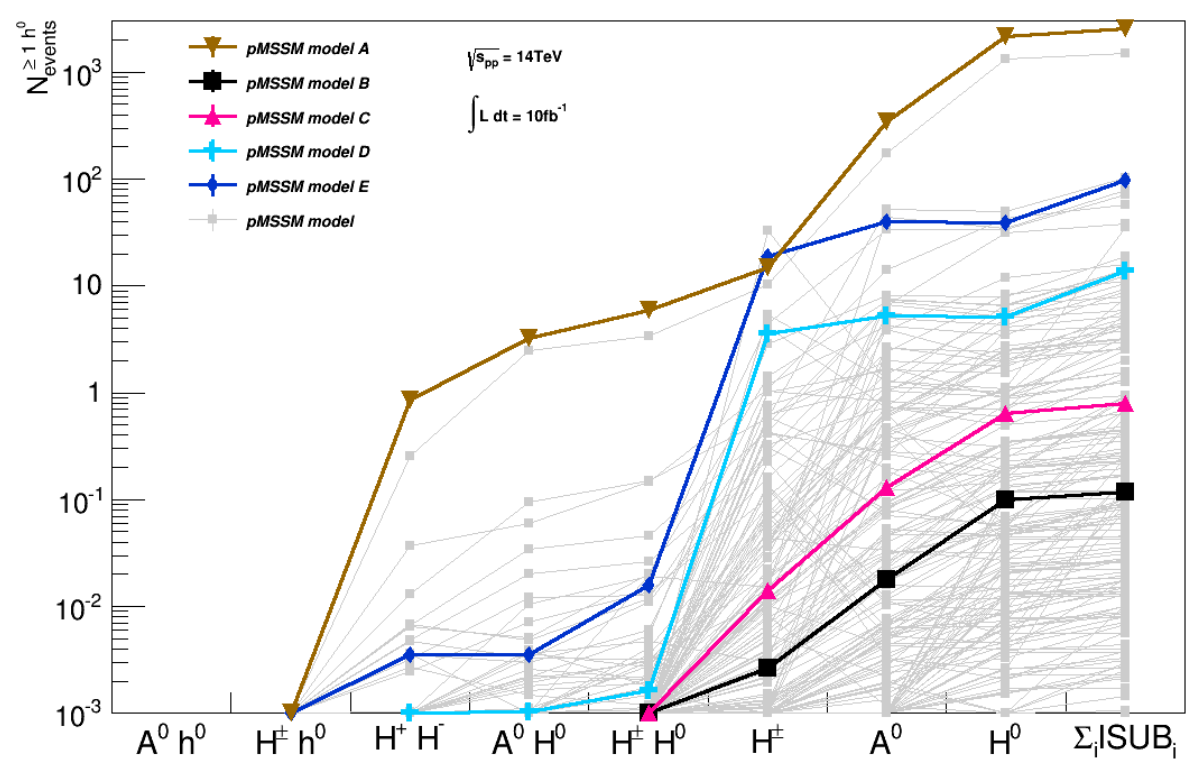

Figure 12. Number of expected events containing at least one $h^{0}$ boson in cascades of heavy-Higgs origin for all candidate models. The horizontal axis indicates the heavy Higgs particles involved in the final states of the primary interaction process.

As can be seen in figure 12, for most models the $h^{0}$ event rates from heavy Higgs production processes is low. This is caused by the decoupling limit. Due to the mass constraint on the lightest Higgs-boson, most models have an $A^{0}$ boson that is much heavier than the $Z$ boson. In this decoupling limit all heavy Higgses are nearly mass degenerate and truly heavy. As a result, the heavy-Higgs production cross sections are relatively small and the $h^{0}$ event rates rather modest. The models with parameters that place them in the decoupling limit only reach a maximum of about $50 h^{0}$ events for single heavy-Higgs production. Exceptions are a couple of models including model A, which have a smaller value for $M_{A}$ and which are therefore less firmly in the decoupling limit. These models also have a relatively small value for $\tan \beta$, which results in a noticeable $H^{0} \rightarrow 2 h^{0}$ branching ratio (see table 3 ) and substantially larger $h^{0}$ event rates beyond 1000 events. Also heavy Higgs production can have an effect on the Standard Model Di-Higgs production rate as discussed in [69].

It must be kept in mind, though, that the heavy Higgs particles are not strictly speaking supersymmetric particles and are therefore not expected to lead to events with a large missing transverse momentum in the detector due to the LSP.

\subsection{Kinematic distributions for Higgs events from SUSY}

Boost of the $\boldsymbol{h}^{0}$ boson. When a supersymmetric particle decays into a $h^{0}$ boson, the mass difference between mother and daughter (initial and final) state can lead to a boost. In hadronic $p p$ collisions the main contribution to $h^{0}$ production by SM processes is expected to be from gluon-gluon fusion, and to a lesser extent from $W W / Z Z$ fusion. A second 


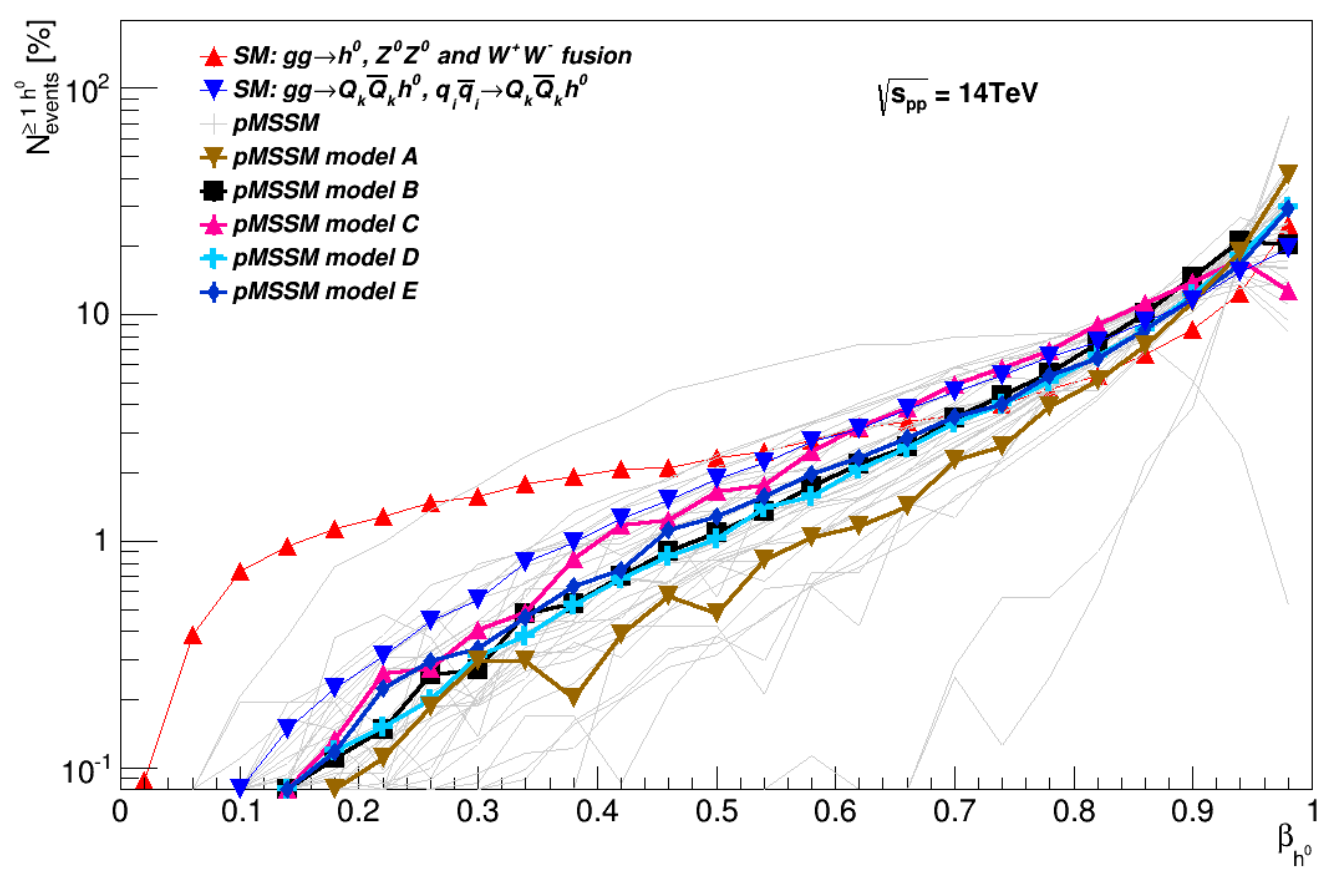

Figure 13. Qualitative distribution (in \%) of the $h^{0}$-boson boost in terms of $\beta_{h^{0}}=v_{h^{0}} / c$ for the main $h^{0}$ hadroproduction processes in the SM (red, blue) and in pMSSM models via SUSY processes (grey). The distributions are each normalised to unity. Only those pMSSM models are presented that predict more than $100 h^{0}$ bosons produced via SUSY processes for $10 \mathrm{fb}^{-1}$.

relevant contribution is expected from associated $t \bar{t} h^{0}$ production, which is expected to lead to $h^{0}$ bosons that are more boosted in view of the larger (top-quark) mass scale in the process. Both processes are shown in figure 13 in order to compare the $h^{0}$-boson boost $\left(\beta_{h^{0}}\right)$ distributions originating from SUSY and SM processes. Due to the larger mass scale of the SUSY processes the $h^{0}$ bosons are on average more boosted, even more than in $t \bar{t} h^{0}$ production. In extreme cases a heavy SUSY particle with mass $>1 \mathrm{TeV}$ decays to a $h^{0}$ boson and a SUSY particle with a mass of $\mathcal{O}(100 \mathrm{GeV})$, leading to a very large boost. As an opposite extreme, we find one case where a squark with $m \approx 1.5 \mathrm{TeV}$ decays to a $\widetilde{\chi}_{3}^{0}$ with $m=1.17 \mathrm{TeV}$, which subsequently decays to a $h^{0}$ and a $\widetilde{\chi}_{1}^{0}$ with $m=1.04 \mathrm{TeV}$. In such compressed scenarios the $h^{0}$ boost is even lower than expected from SM processes.

Missing transverse momentum. Figure 14 shows the missing transverse momentum distributions from SUSY processes for the selected pMSSM models. The generated events are normalized to an integrated luminosity of $10 \mathrm{fb}^{-1}$. All models have on average large missing transverse momentum up to several $100 \mathrm{GeV}$, permitting the introduction of selection cuts of $100-200 \mathrm{GeV}$ in order to reduce backgrounds from SM processes. The production of heavy Higgs bosons is not considered. Model $A$ has low missing transverse momentum since the $h^{0}$ boson originates from a heavy $H^{0}$ boson, which does not decay to the LSP. 


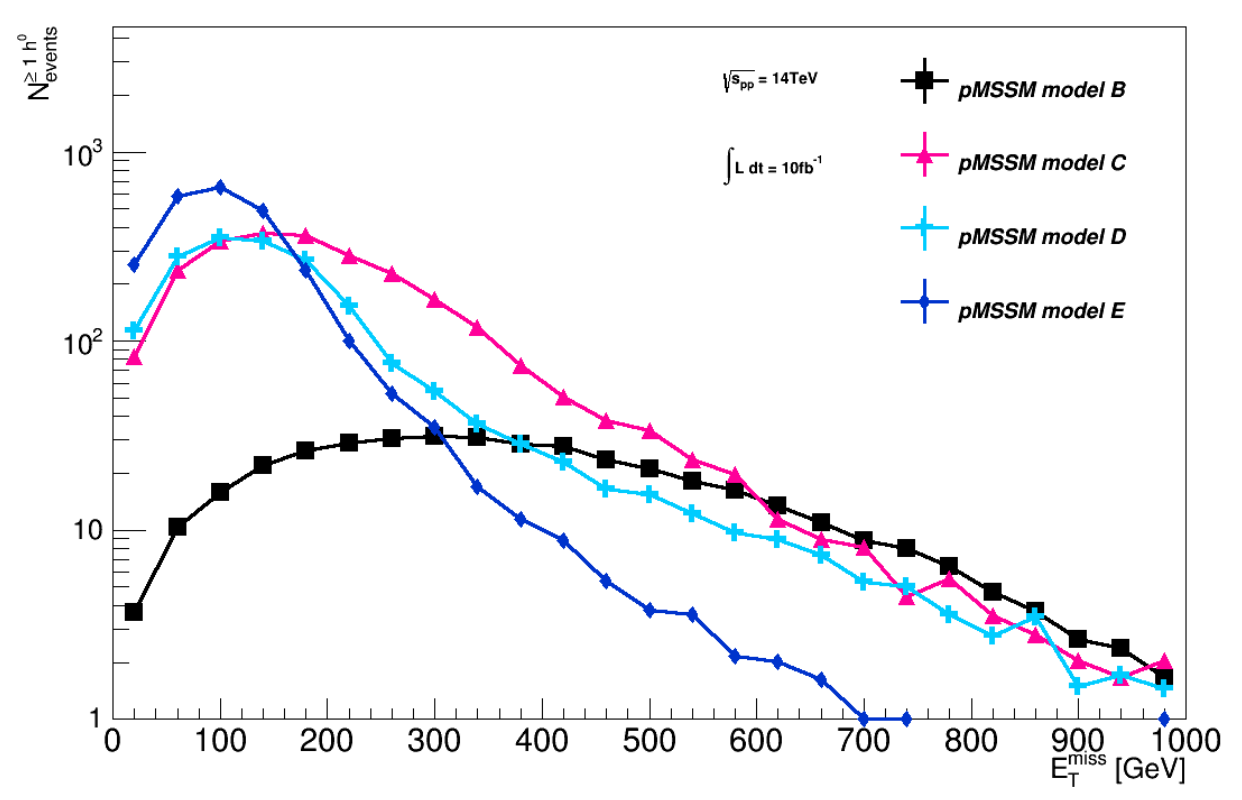

Figure 14. Number of expected events containing at least one $h^{0}$ boson as a function of missing transverse energy $\mathrm{E}_{\mathrm{T}}^{\mathrm{miss}}$ in cascades of supersymmetric origin (i.e. without the production of heavy Higgs bosons).

Final states with $\boldsymbol{h}^{\mathbf{0}}$ bosons. After the detector response is simulated with DELPHES, the final states are determined. Selection cuts are applied, requiring the leptons, i.e. electrons or muons, to have a transverse momentum of at least $20 \mathrm{GeV}$. For the jets this lower limit is chosen to be $50 \mathrm{GeV}$. B-jets and hadronic tau decays are counted as jets. Both leptons and jets are only considered if they are located within the pseudorapidity ${ }^{4}$ region of $\left|\eta^{e, \mu, j e t}\right|<2.5$. In addition to the overlay removal that is automatically performed in DELPHES, a stricter overlap removal of $\Delta R_{a, b}>0.6$ is applied. ${ }^{5}$ The generated events are again normalised to an integrated luminosity of $10 \mathrm{fb}^{-1}$.

The lepton and jet multiplicities for events requiring a missing transverse momentum of at least $100 \mathrm{GeV}$ are shown in figures 15,16 and 17 . The most populated channels for single Higgs production are the channels that contain $\geq 1-4$ jets, with close to 1000 events, and mono-higgs ${ }^{6}$ production, with up to 200 events for $10 \mathrm{fb}^{-1}$. In some cases very high jet multiplicities can occur, as can be seen in figure 17. Channels with one lepton lead to $\sim 100$ events and channels with two leptons to less than 10 events. Higher lepton multiplicities are not important for $h^{0}$ production. Di-Higgs and tri-Higgs production is dominantly found in channels with $\geq 2$ jets. Another notable feature is that the production of neutralino pairs can lead to events with two Higgs bosons, missing transverse momentum and nothing else, i.e. no leptons and no jets.

\footnotetext{
${ }^{4} \eta=-\ln \left[\tan \left(\frac{\theta}{2}\right)\right]$ in terms of the polar angle $\theta$ w.r.t. the beam axis.

${ }^{5} \Delta R_{a, b}=\sqrt{(\Delta \eta)_{a, b}^{2}+(\Delta \phi)_{a, b}^{2}}$ in terms of the pseudorapidity difference $\Delta \eta$ and the difference in azimuthal angle $\Delta \phi$ between the objects (leptons/jets) $a$ and $b$.

${ }^{6}$ The 0-lepton, 0 -jet channel, which is dominated by neutralino-pair production.
} 


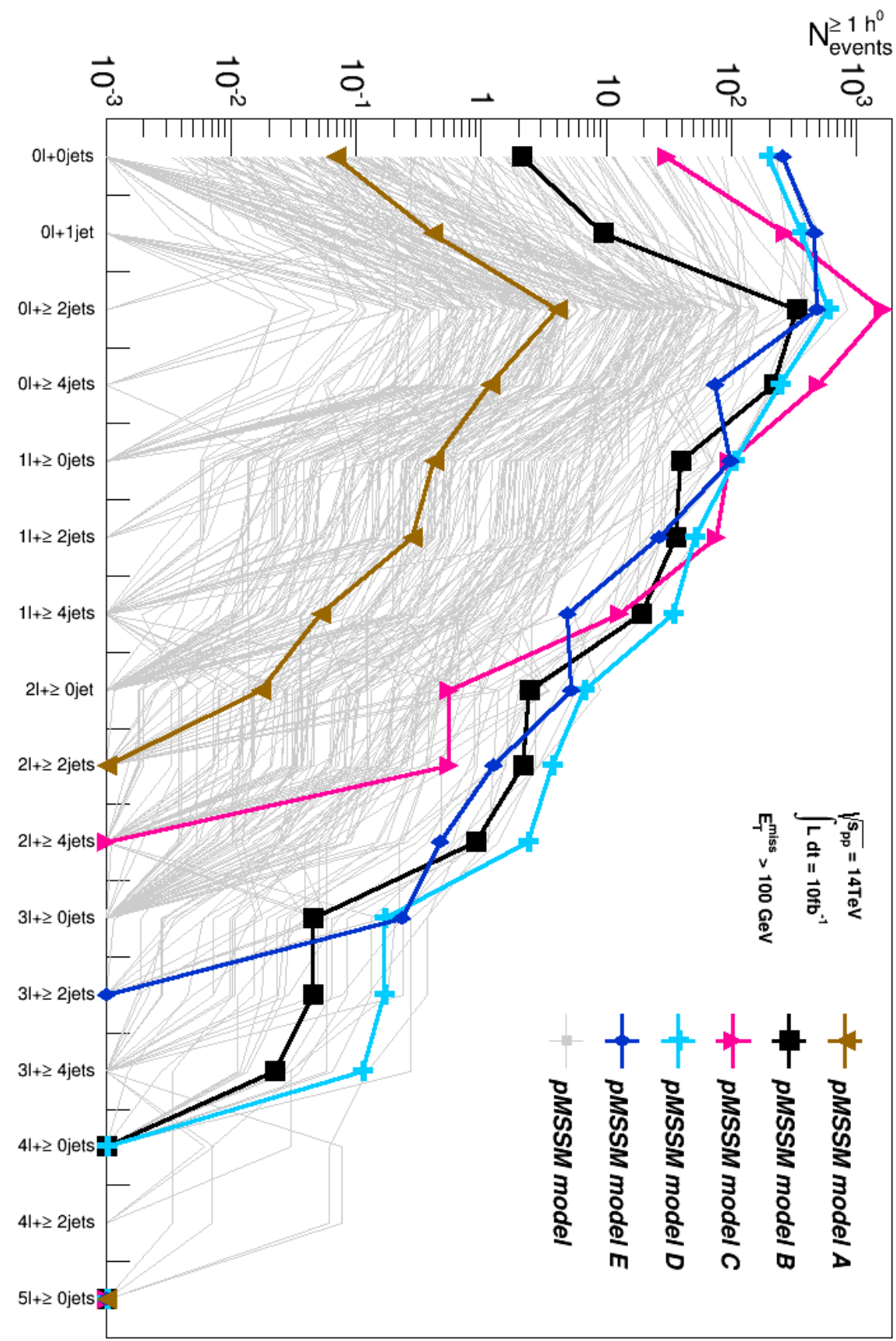

Figure 15. Number of expected events containing at least one $h^{0}$ boson in cascades of supersymmetric origin with $\mathrm{E}_{\mathrm{T}}^{\mathrm{miss}}>100 \mathrm{GeV}$. The event rates are split up according to specific combinations of lepton and jet multiplicities. 


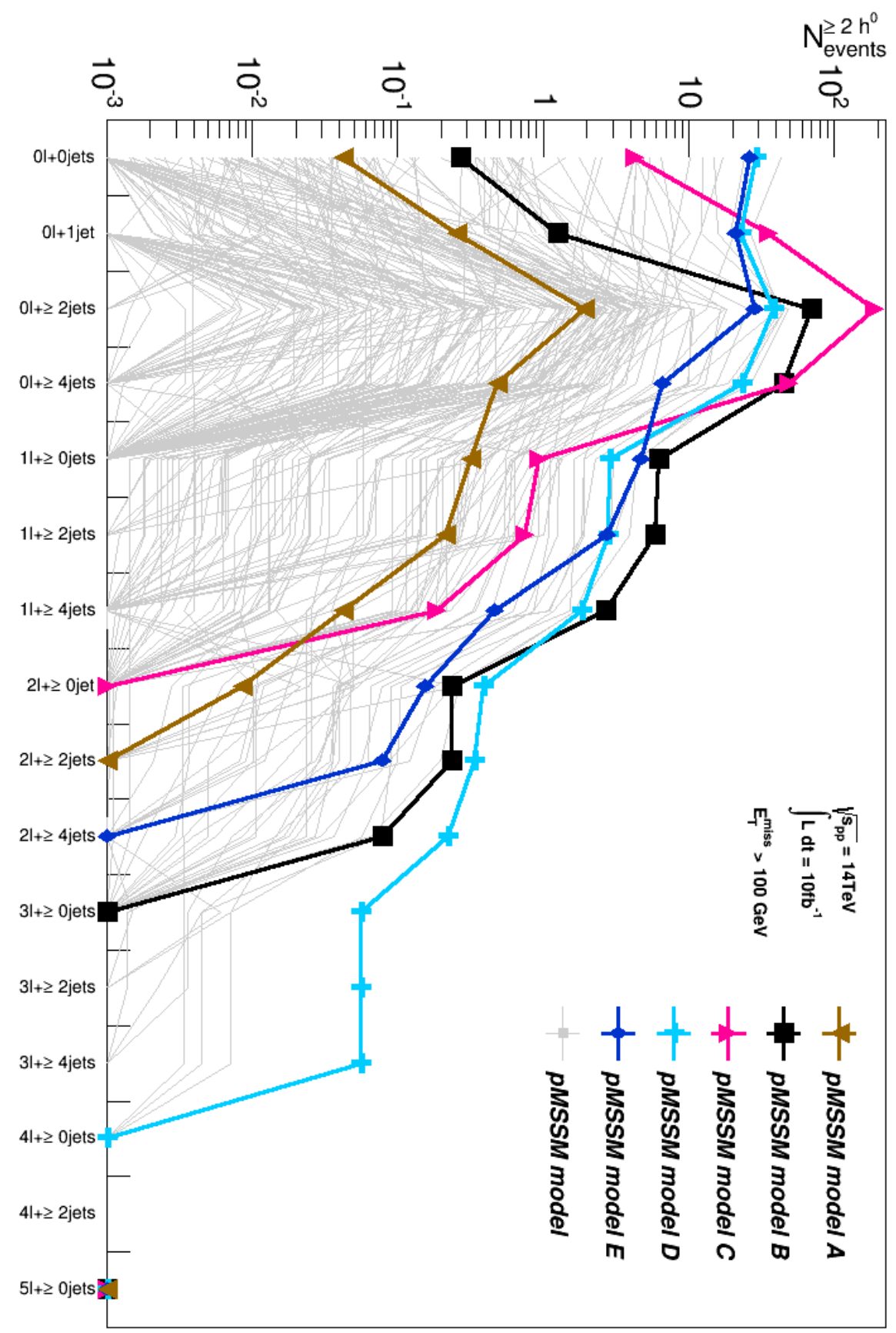

Figure 16. Number of expected events containing at least two $h^{0}$ bosons in cascades of supersymmetric origin with $\mathrm{E}_{\mathrm{T}}^{\mathrm{miss}}>100 \mathrm{GeV}$. The event rates are split up according to specific combinations of lepton and jet multiplicities. 

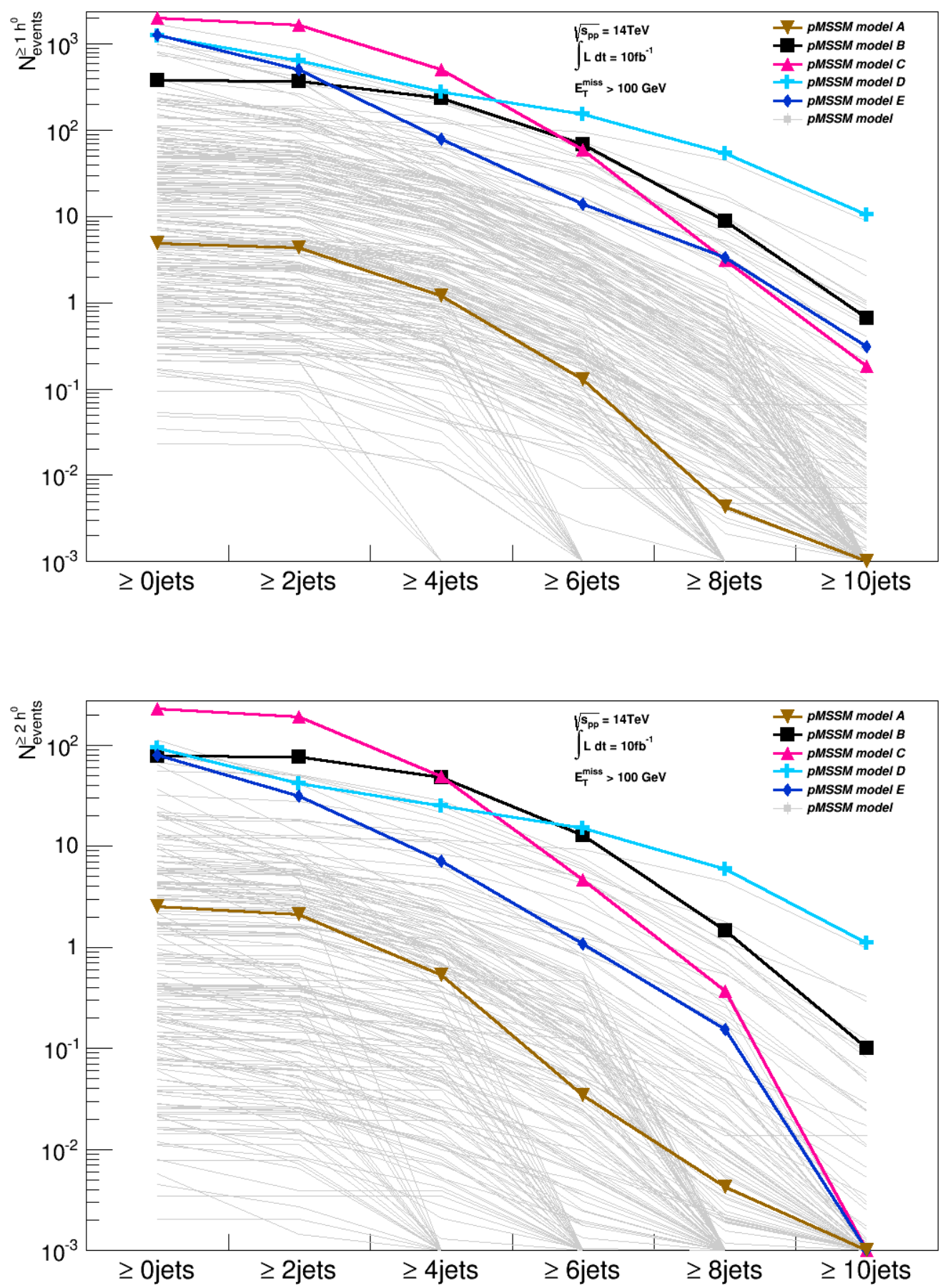

Figure 17. Number of expected events containing at least one $h^{0}$ boson (upper figure) or two $h^{0}$ bosons (lower figure) in cascades of supersymmetric origin with $\mathrm{E}_{\mathrm{T}}^{\mathrm{miss}}>100 \mathrm{GeV}$. The event rates are split up according to specific jet multiplicities. 


\section{Conclusion}

We have systematically investigated the possibilities to produce a $125 \mathrm{GeV}$ Higgs boson $\left(h^{0}\right)$ via SUSY processes within the phenomenological MSSM (pMSSM). We find the following interesting features:

- Given global constraints on the pMSSM, it is possible to produce Higgs events with a large rate in the upcoming LHC data at the increased centre-of-mass energy. We have found valid pMSSM models that could produce more than 3000 Higgs, 300 di-Higgs and/or 20 tri-Higgs events already with an integrated luminosity of $10 \mathrm{fb}^{-1}$.

- A relation is observed between large Higgs-production rates via squark decays to heavy neutralinos and inherent difficulties to exclude such models in conventional (non-Higgs) LHC searches. This is caused by the fact that Higgs production requires a less compressed neutralino mass spectrum, which can bring the heavy neutralinos closer to the lowest-lying squark states, thereby reducing the available amount of energy for additional jets.

- In some models Higgs production is significant for almost all important SUSY production processes, which can have large repercussions on SM Higgs studies and SUSY searches.

- Higgs production via SUSY processes might significantly enhance the event rates for SM Higgs and di-Higgs searches, especially in final states with missing transverse momentum. The allowed SUSY production rates can be reduced by upcoming (negative) SUSY searches at higher LHC energies, especially if new dedicated searches for events with $h^{0}$ bosons and missing transverse momentum are performed.

- Higgs production processes can likewise be of importance for a SUSY discovery via "Higgs tagging". We found that the different SUSY production channels can lead to a large variety of interesting event topologies and kinematics. Of special interest are multi-jet channels with up to three Higgs bosons, "mono-Higgs" channels with up to two Higgs bosons, one-lepton channels with one Higgs boson and Higgs production in association with top or b-quarks, all with a sizeable amount of missing transverse momentum. The list is completed by searches for heavy Higgs bosons decaying directly or via neutralinos/charginos into $h^{0}$ bosons. 


\section{A Selected illustrative figures}
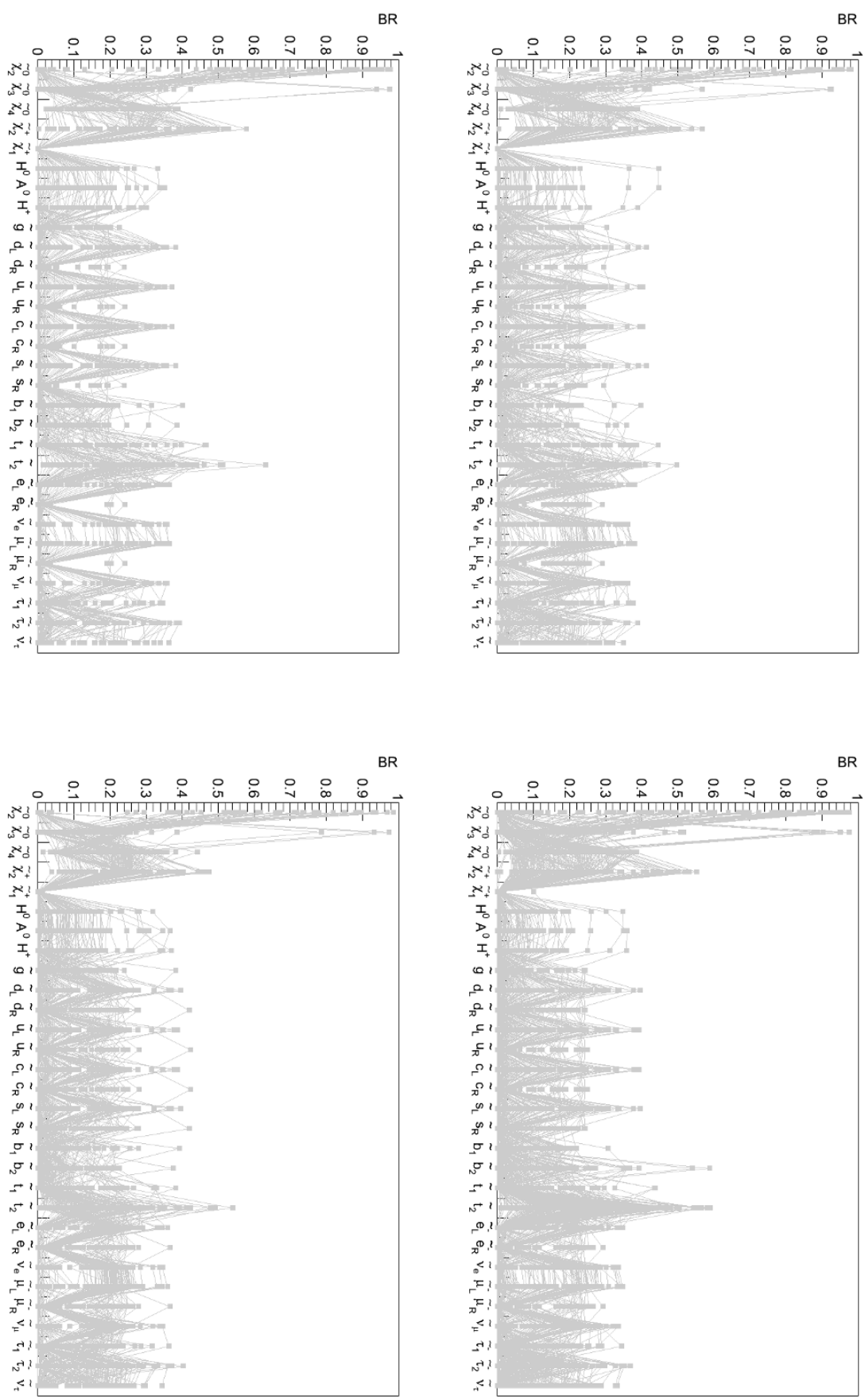

Figure 18. Branching ratios into $h^{0}$ for all MSSM particles. The plots show set B with the width of the Gaussian particle filter set to $10 \%$ (upper left), 25\% (upper right) and 40\% (lower left) of the full extent of the parameter space in each dimension. The lower right figure shows for comparison set A, which has a different Higgs branching ratio requirement. 

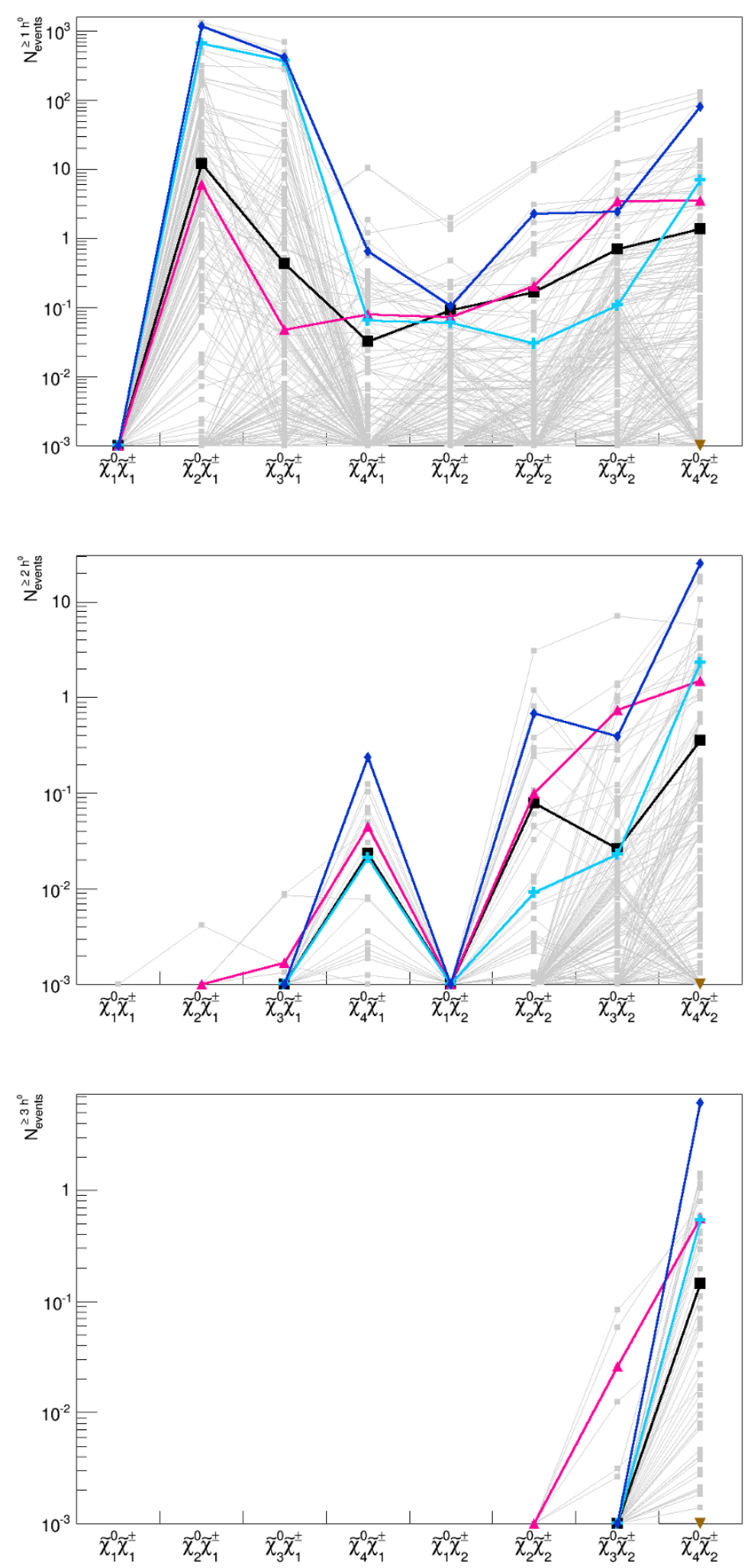

Figure 19. Number of expected events containing at least one (upper), two (middle) or three (lower figure) $h^{0}$ boson(s) produced in cascades of supersymmetric origin. The horizontal axis indicates the supersymmetric neutralino-chargino final states of the primary interaction process. 

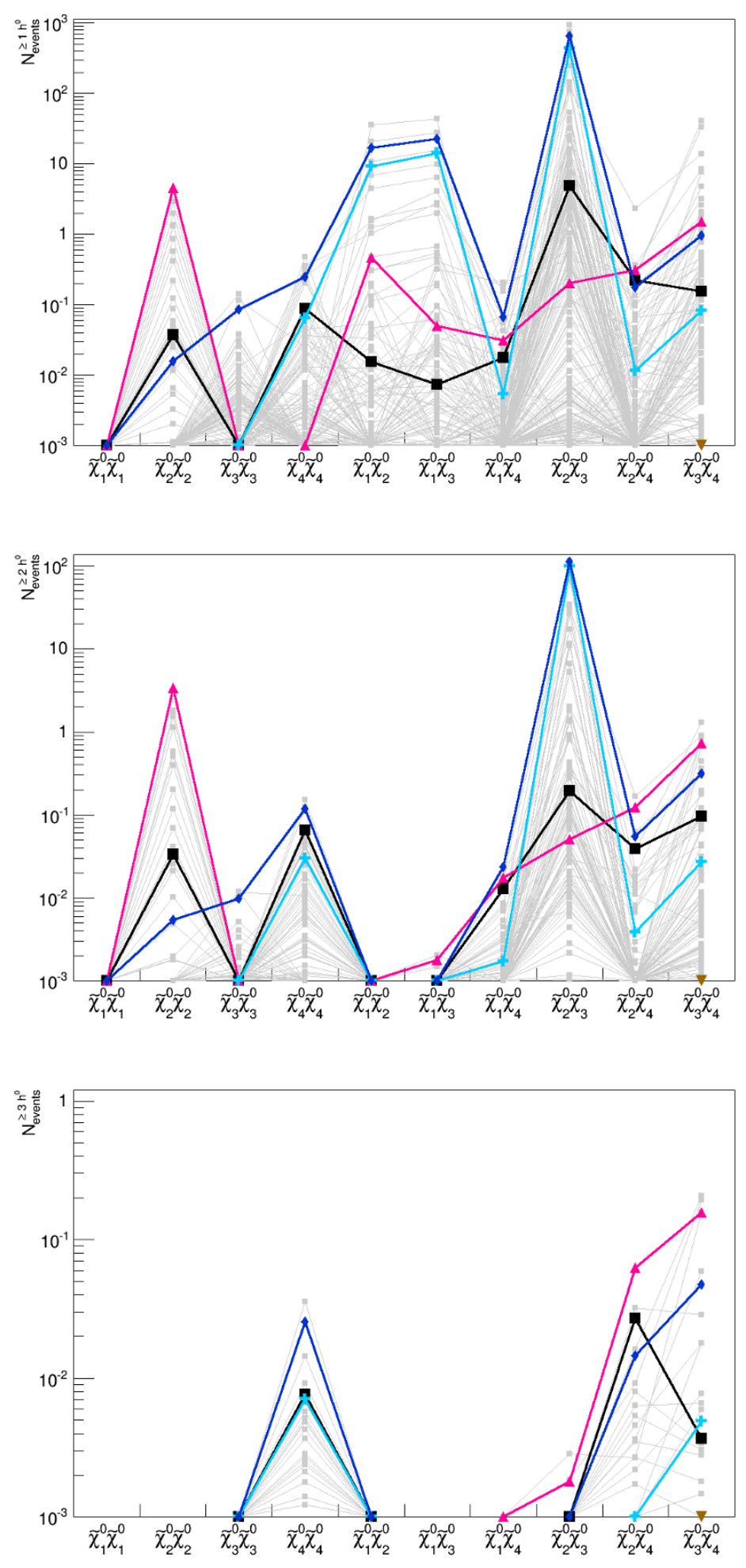

Figure 20. Number of expected events containing at least one (upper), two (middle) or three (lower figure) $h^{0}$ boson(s) produced in cascades of supersymmetric origin. The horizontal axis indicates the supersymmetric neutralino-neutralino final states of the primary interaction process. 

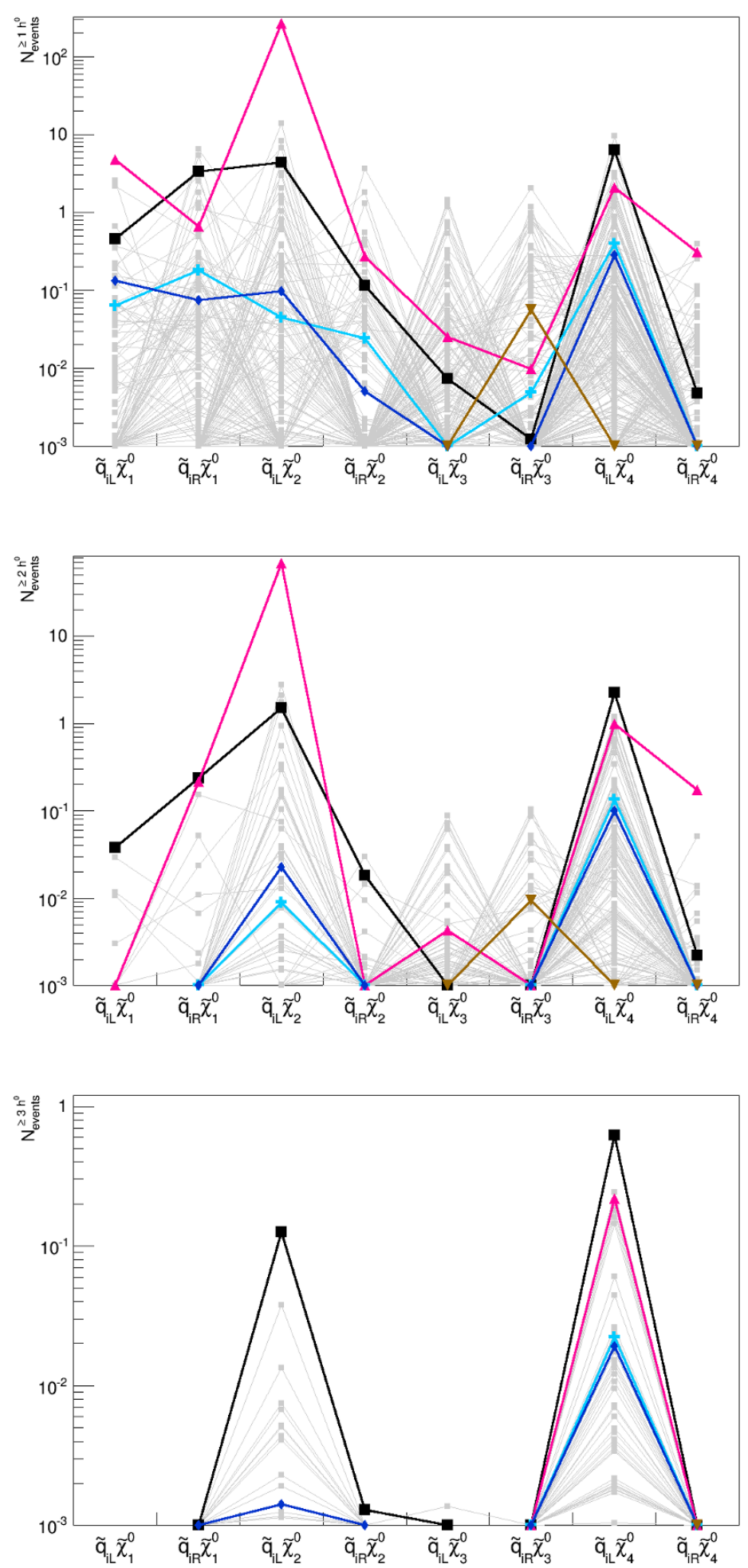

Figure 21. Number of expected events containing at least one (upper), two (middle) or three (lower figure) $h^{0}$ boson(s) produced in cascades of supersymmetric origin. The horizontal axis indicates the supersymmetric squark-neutralino final states of the primary interaction process. 

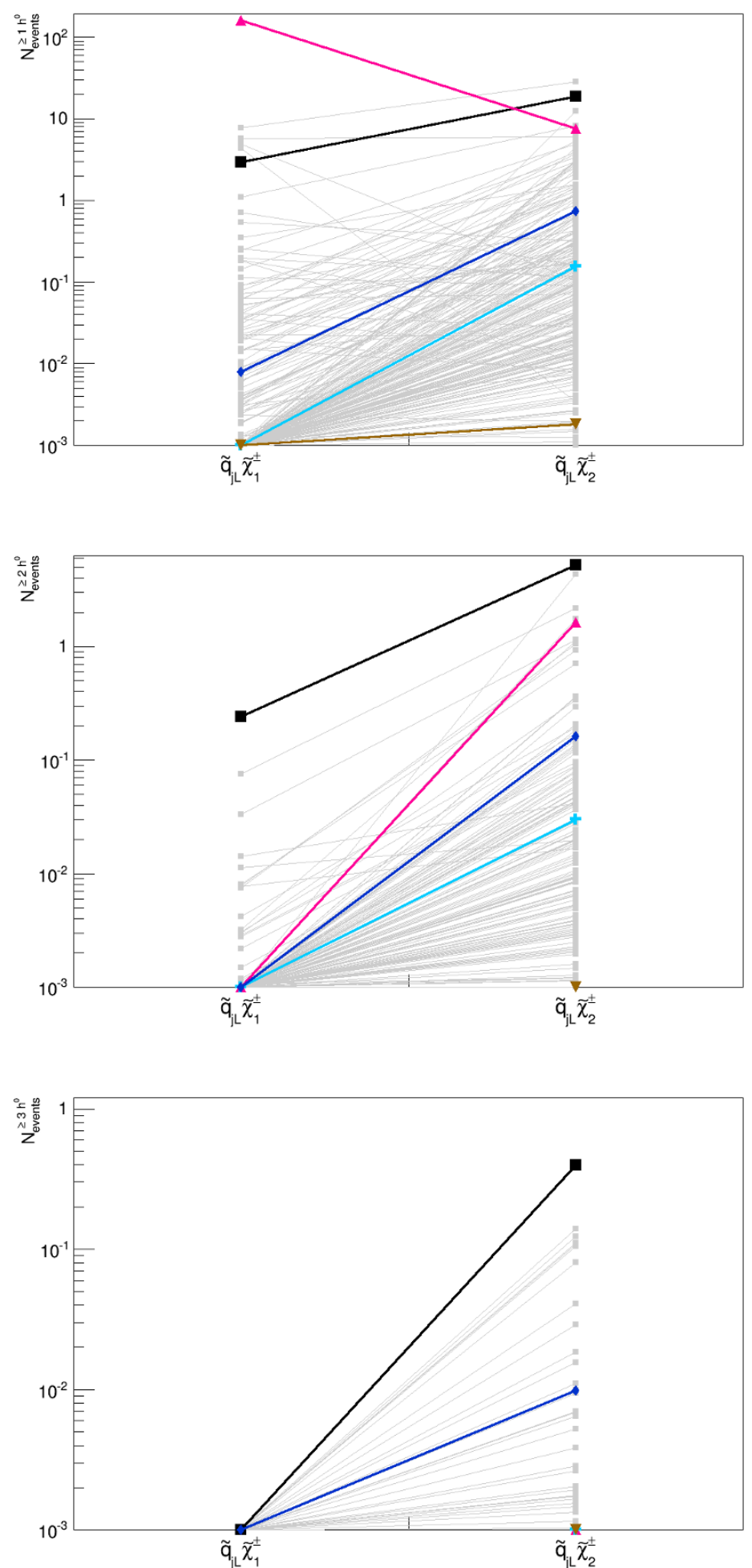

Figure 22. Number of expected events containing at least one (upper), two (middle) or three (lower figure) $h^{0}$ boson(s) produced in cascades of supersymmetric origin. The horizontal axis indicates the supersymmetric left-handed-squark-chargino final states of the primary interaction process. 

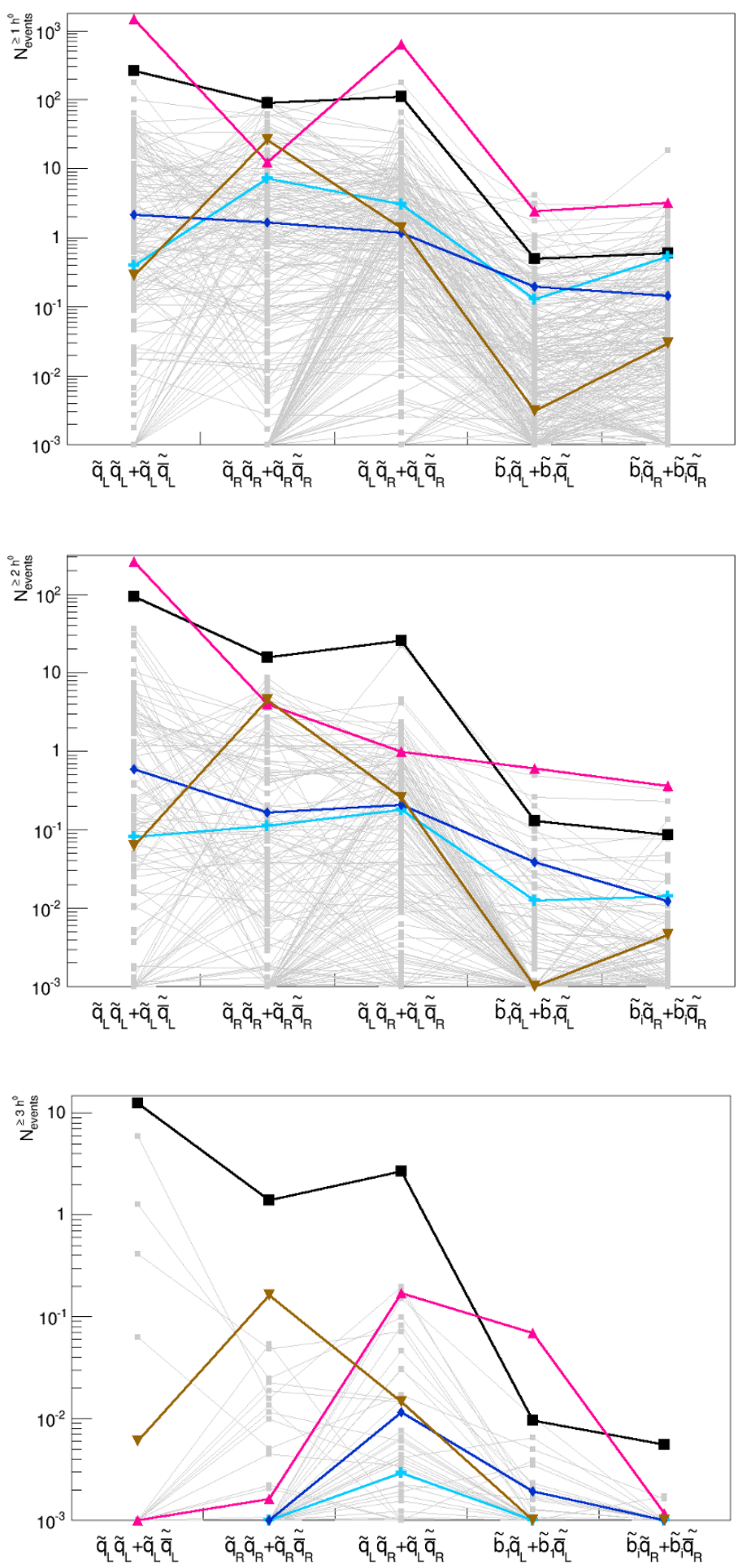

Figure 23. Number of expected events containing at least one (upper), two (middle) or three (lower figure) $h^{0}$ boson(s) produced in cascades of supersymmetric origin. The horizontal axis indicates the supersymmetric squark-(anti)squark final states of the primary interaction process, where at least one of the squarks is of the 1st/2nd generation. 

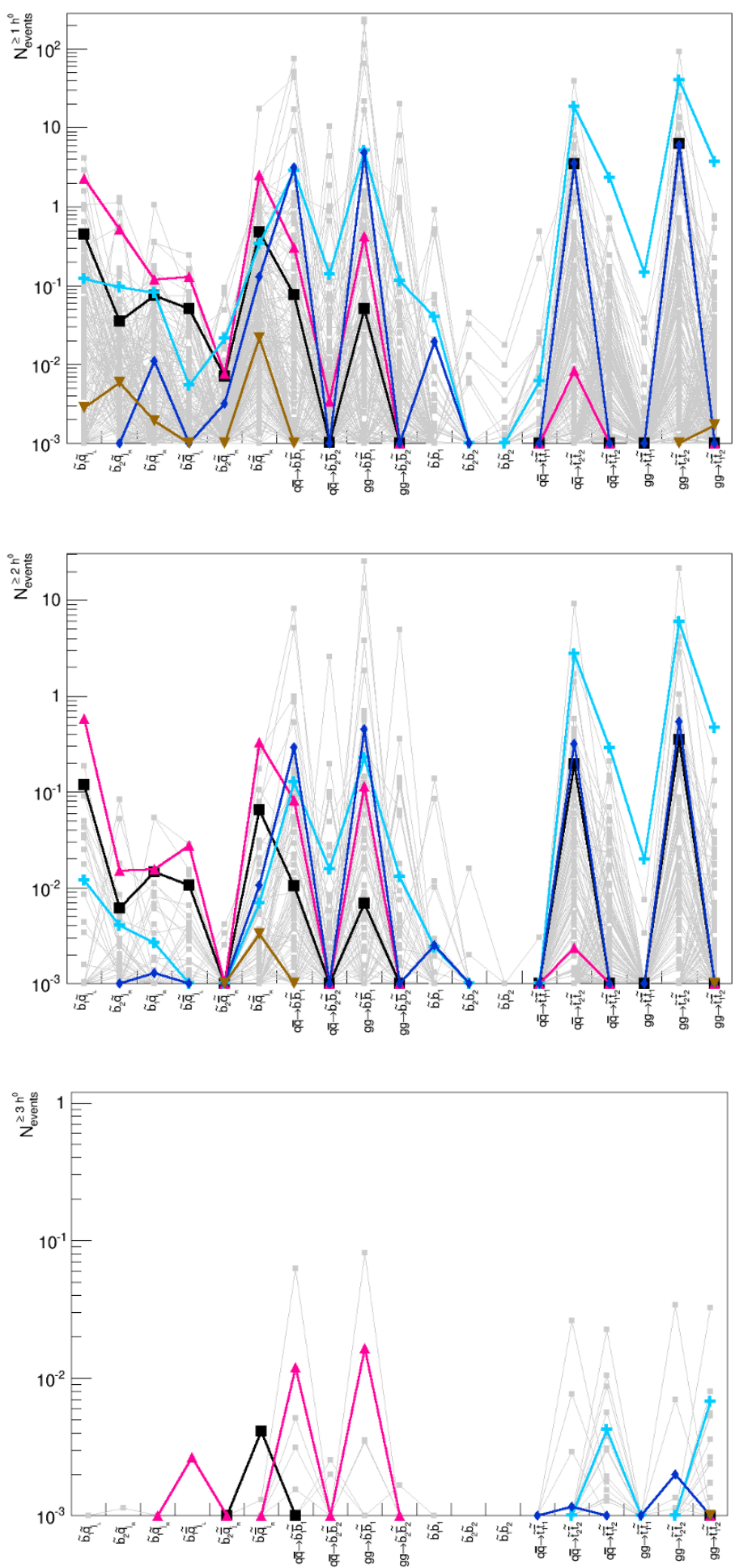

Figure 24. Number of expected events containing at least one (upper), two (middle) or three (lower figure) $h^{0}$ boson(s) produced in cascades of supersymmetric origin. The horizontal axis indicates the supersymmetric squark-(anti)squark final states of the primary interaction process, where at least one of the squarks is of the 3rd generation. 

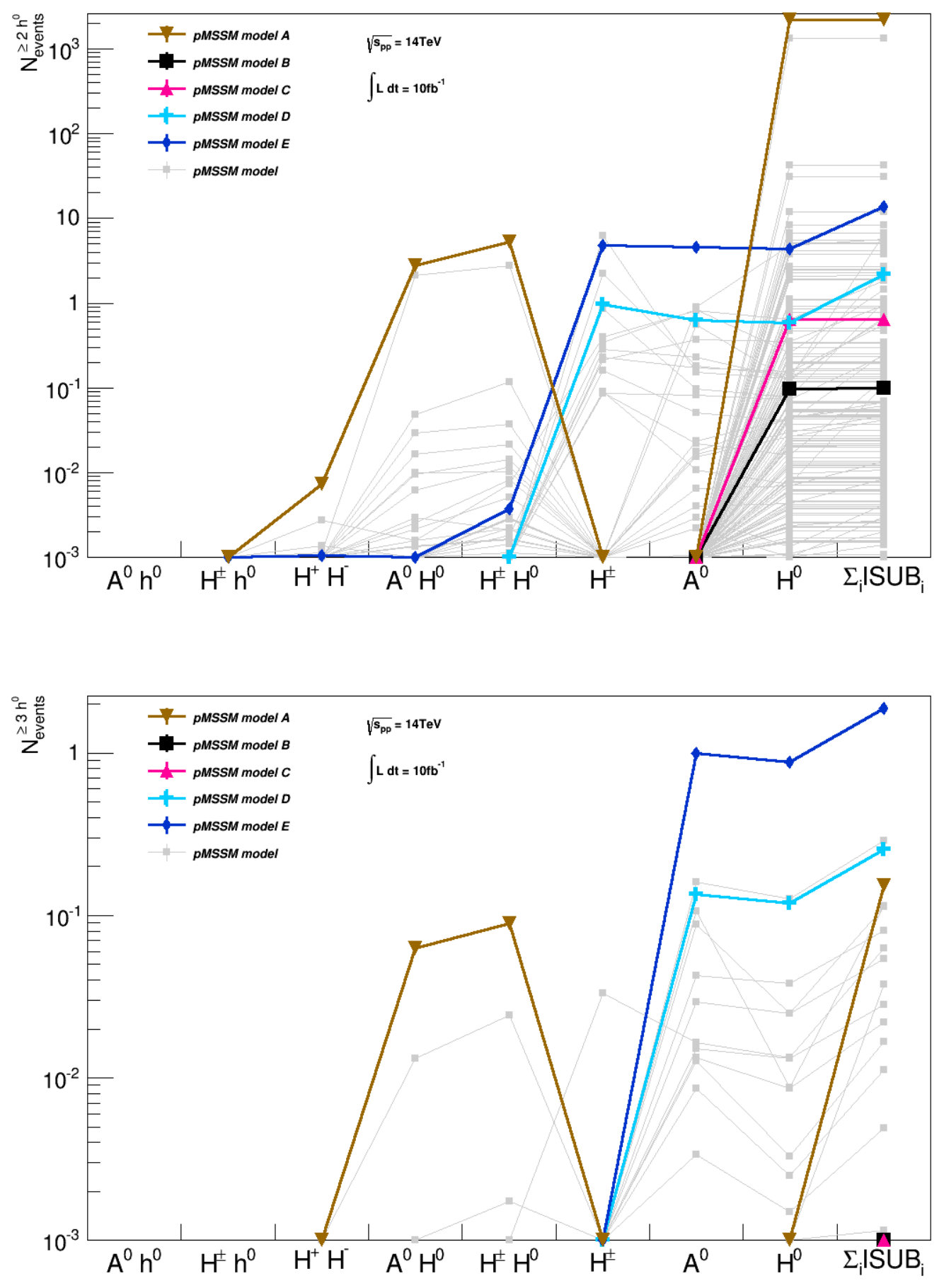

Figure 25. Number of expected events containing at least two (upper) or three (lower) $h^{0}$ bosons in cascades of heavy-Higgs $\left(H^{0}, A^{0}\right.$ and $\left.H^{ \pm}\right)$origin. The horizontal axis indicates the heavy Higgs particles involved in the final states of the primary interaction process. 
Open Access. This article is distributed under the terms of the Creative Commons Attribution License (CC-BY 4.0), which permits any use, distribution and reproduction in any medium, provided the original author(s) and source are credited.

\section{References}

[1] ATLAS collaboration, Observation of a new particle in the search for the Standard Model Higgs boson with the ATLAS detector at the LHC, Phys. Lett. B 716 (2012) 1 [arXiv: 1207.7214] [INSPIRE].

[2] CMS collaboration, Observation of a new boson at a mass of $125 \mathrm{GeV}$ with the CMS experiment at the LHC, Phys. Lett. B 716 (2012) 30 [arXiv:1207.7235] [INSPIRE].

[3] H. Miyazawa, Baryon Number Changing Currents, Prog. Theor. Phys. 36 (1966) 1266 [INSPIRE].

[4] P. Ramond, Dual Theory for Free Fermions, Phys. Rev. D 3 (1971) 2415 [inSPIRE].

[5] Y. Golfand and E.P. Likhtman, Extension of the Algebra of Poincaré Group Generators and Violation of p Invariance, JETP Lett. 13 (1971) 323 [INSPIRE].

[6] A. Neveu and J.H. Schwarz, Factorizable dual model of pions, Nucl. Phys. B 31 (1971) 86 [INSPIRE].

[7] A. Neveu and J.H. Schwarz, Quark Model of Dual Pions, Phys. Rev. D 4 (1971) 1109 [INSPIRE].

[8] J.-L. Gervais and B. Sakita, Field Theory Interpretation of Supergauges in Dual Models, Nucl. Phys. B 34 (1971) 632 [inSPIRE].

[9] D.V. Volkov and V.P. Akulov, Is the Neutrino a Goldstone Particle?, Phys. Lett. B 46 (1973) 109 [INSPIRE].

[10] J. Wess and B. Zumino, A Lagrangian Model Invariant Under Supergauge Transformations, Phys. Lett. B 49 (1974) 52 [INSPIRE].

[11] J. Wess and B. Zumino, Supergauge Transformations in Four-Dimensions, Nucl. Phys. B 70 (1974) 39 [INSPIRE].

[12] P. Fayet, Supersymmetry and Weak, Electromagnetic and Strong Interactions, Phys. Lett. B 64 (1976) 159 [INSPIRE].

[13] P. Fayet, Spontaneously Broken Supersymmetric Theories of Weak, Electromagnetic and Strong Interactions, Phys. Lett. B 69 (1977) 489 [INSPIRE].

[14] G.R. Farrar and P. Fayet, Phenomenology of the Production, Decay and Detection of New Hadronic States Associated with Supersymmetry, Phys. Lett. B 76 (1978) 575 [INSPIRE].

[15] P. Fayet, Relations Between the Masses of the Superpartners of Leptons and Quarks, the Goldstino Couplings and the Neutral Currents, Phys. Lett. B 84 (1979) 416 [INSPIRE].

[16] S. Dimopoulos and H. Georgi, Softly Broken Supersymmetry and SU(5), Nucl. Phys. B 193 (1981) 150 [INSPIRE].

[17] H. Goldberg, Constraint on the Photino Mass from Cosmology, Phys. Rev. Lett. 50 (1983) 1419 [INSPIRE].

[18] J.R. Ellis, J.S. Hagelin, D.V. Nanopoulos, K.A. Olive and M. Srednicki, Supersymmetric Relics from the Big Bang, Nucl. Phys. B 238 (1984) 453 [INSPIRE]. 
[19] E. Witten, Dynamical breaking of supersymmetry, Nucl. Phys. B 188 (1981) 513 [INSPIRE].

[20] M. Dine, W. Fischler and M. Srednicki, Supersymmetric Technicolor, Nucl. Phys. B 189 (1981) 575 [INSPIRE].

[21] S. Dimopoulos and S. Raby, Supercolor, Nucl. Phys. B 192 (1981) 353 [inSPIRE].

[22] N. Sakai, Naturalness in Supersymmetric Guts, Z. Phys. C 11 (1981) 153.

[23] R.K. Kaul and P. Majumdar, Cancellation of Quadratically Divergent Mass Corrections in Globally Supersymmetric Spontaneously Broken Gauge Theories, Nucl. Phys. B 199 (1982) 36 [INSPIRE].

[24] A. Djouadi, J.-L. Kneur and G. Moultaka, SuSpect: A Fortran code for the supersymmetric and Higgs particle spectrum in the MSSM, Comput. Phys. Commun. 176 (2007) 426 [hep-ph/0211331] [INSPIRE].

[25] C.F. Berger, J.S. Gainer, J.L. Hewett and T.G. Rizzo, Supersymmetry Without Prejudice, JHEP 02 (2009) 023 [arXiv: 0812.0980] [INSPIRE].

[26] D. Ghosh, M. Guchait and D. Sengupta, Higgs Signal in Chargino-Neutralino Production at the LHC, Eur. Phys. J. C 72 (2012) 2141 [arXiv:1202.4937] [InSPIRE].

[27] K. Howe and P. Saraswat, Excess Higgs Production in Neutralino Decays, JHEP 10 (2012) 065 [arXiv: 1208.1542] [INSPIRE].

[28] S. Gori, P. Schwaller and C.E.M. Wagner, Search for Higgs Bosons in SUSY Cascade Decays and Neutralino Dark Matter, Phys. Rev. D 83 (2011) 115022 [arXiv:1103.4138] [InSPIRE].

[29] D. Ghosh, Boosted dibosons from mixed heavy top squarks, Phys. Rev. D 88 (2013) 115013 [arXiv: 1308.0320] [INSPIRE].

[30] G.D. Kribs, A. Martin, T.S. Roy and M. Spannowsky, Discovering the Higgs Boson in New Physics Events using Jet Substructure, Phys. Rev. D 81 (2010) 111501 [arXiv:0912.4731] [INSPIRE].

[31] G.D. Kribs, A. Martin, T.S. Roy and M. Spannowsky, Discovering Higgs Bosons of the MSSM using Jet Substructure, Phys. Rev. D 82 (2010) 095012 [arXiv:1006.1656] [INSPIRE].

[32] G.D. Kribs, A. Martin and T.S. Roy, Higgs boson discovery through top-partners decays using jet substructure, Phys. Rev. D 84 (2011) 095024 [arXiv:1012.2866] [INSPIRE].

[33] P. Byakti and D. Ghosh, Magic Messengers in Gauge Mediation and signal for $125 \mathrm{GeV}$ boosted Higgs boson, Phys. Rev. D 86 (2012) 095027 [arXiv: 1204.0415] [INSPIRE].

[34] ATLAS collaboration, Search for direct production of charginos and neutralinos decaying via the $125 \mathrm{GeV}$ Higgs boson in $\sqrt{s}=8 \mathrm{TeV}$ pp collisions with the ATLAS detector, ATLAS-CONF-2014-062 (2014).

[35] CMS collaboration, Searches for electroweak neutralino and chargino production in channels with Higgs, $Z$ and $W$ bosons in pp collisions at 8 TeV, Phys. Rev. D 90 (2014) 092007 [arXiv: 1409.3168] [INSPIRE].

[36] CMS collaboration, Searches for electroweak production of charginos, neutralinos and sleptons decaying to leptons and $W, Z$ and Higgs bosons in pp collisions at $8 \mathrm{TeV}$, Eur. Phys. J. C 74 (2014) 3036 [arXiv:1405.7570] [INSPIRE].

[37] CMS collaboration, Search for top-squark pairs decaying into Higgs or $Z$ bosons in pp collisions at $\sqrt{s}=8 \mathrm{TeV}$, Phys. Lett. B 736 (2014) 371 [arXiv:1405.3886] [InSPIRE]. 
[38] CMS collaboration, Search for top squark and higgsino production using diphoton Higgs boson decays, Phys. Rev. Lett. 112 (2014) 161802 [arXiv:1312.3310] [INSPIRE].

[39] S.P. Martin, A Supersymmetry primer, Adv. Ser. Direct. High Energy Phys. 21 (2010) 1 [hep-ph/9709356] [INSPIRE].

[40] A. Arbey, M. Battaglia and F. Mahmoudi, Higgs Production in Neutralino Decays in the MSSM - The LHC and a Future $e^{+} e^{-}$Collider, Eur. Phys. J. C 75 (2015) 108 [arXiv: 1212.6865] [INSPIRE].

[41] A. Djouadi, The Anatomy of electro-weak symmetry breaking. II. The Higgs bosons in the minimal supersymmetric model, Phys. Rept. 459 (2008) 1 [hep-ph/0503173] [INSPIRE].

[42] P.Z. Skandsr et al., SUSY Les Houches accord: interfacing SUSY spectrum calculators, decay packages and event generators, JHEP 07 (2004) 036 [hep-ph/0311123] [INSPIRE].

[43] ATLAS collaboration, Measurement of the Higgs boson mass from the $H \rightarrow \gamma \gamma$ and $H \rightarrow Z Z^{*} \rightarrow 4 \ell$ channels with the ATLAS detector using $25 \mathrm{fb}^{-1}$ of pp collision data, Phys. Rev. D 90 (2014) 052004 [arXiv: 1406.3827] [INSPIRE].

[44] CMS collaboration, Precise determination of the mass of the Higgs boson and tests of compatibility of its couplings with the standard model predictions using proton collisions at 7 and $8 \mathrm{TeV}$, CERN-PH-EP-2014-288, CMS-HIG-14-009-003 (2014) [arXiv:1412.8662].

[45] N. Gordon, D. Salmond and A.F. Smith, Novel approach to nonlinear/non-Gaussian Bayesian state estimation, IEE Proc. F Radar Signal Process. 140 (1993) 107.

[46] G. Bélanger, F. Boudjema, A. Pukhov and A. Semenov, MicrOMEGAs: A Program for calculating the relic density in the MSSM, Comput. Phys. Commun. 149 (2002) 103 [hep-ph/0112278] [INSPIRE].

[47] WMAP collaboration, E. Komatsu et al., Five-Year Wilkinson Microwave Anisotropy Probe (WMAP) Observations: Cosmological Interpretation, Astrophys. J. Suppl. 180 (2009) 330 [arXiv: 0803.0547] [INSPIRE].

[48] Planck collaboration, P.A.R. Ade et al., Planck 2013 results. XVI. Cosmological parameters, Astron. Astrophys. 571 (2014) A16 [arXiv:1303.5076] [INSPIRE].

[49] LUX collaboration, D.S. Akerib et al., First results from the LUX dark matter experiment at the Sanford Underground Research Facility, Phys. Rev. Lett. 112 (2014) 091303 [arXiv: 1310.8214] [INSPIRE].

[50] G. Bélanger, M. Kakizaki, E.K. Park, S. Kraml and A. Pukhov, Light mixed sneutrinos as thermal dark matter, JCAP 11 (2010) 017 [arXiv:1008.0580] [INSPIRE].

[51] LHCb collaboration, Measurement of the $B_{s}^{0} \rightarrow \mu^{+} \mu^{-}$branching fraction and search for $B^{0} \rightarrow \mu^{+} \mu^{-}$decays at the LHCb experiment, Phys. Rev. Lett. 111 (2013) 101805 [arXiv: 1307.5024] [INSPIRE].

[52] CMS collaboration, Measurement of the B(s) to $\mu^{+} \mu^{-}$branching fraction and search for B0 to $\mu^{+} \mu^{-}$with the CMS Experiment, Phys. Rev. Lett. 111 (2013) 101804 [arXiv:1307.5025] [INSPIRE].

[53] ATLAS collaboration, Search for neutral Higgs bosons of the minimal supersymmetric standard model in pp collisions at $\sqrt{s}=8 \mathrm{TeV}$ with the ATLAS detector, JHEP 11 (2014) 056 [arXiv: 1409.6064] [INSPIRE]. 
[54] CMS collaboration, Search for neutral MSSM Higgs bosons decaying to a pair of tau leptons in pp collisions, JHEP 10 (2014) 160 [arXiv:1408.3316] [INSPIRE].

[55] T. Sjöstrand, S. Mrenna and P.Z. Skands, PYTHIA 6.4 Physics and Manual, JHEP 05 (2006) 026 [hep-ph/0603175] [inSPIRE].

[56] DELPHES 3 collaboration, J. de Favereau et al., DELPHES 3, A modular framework for fast simulation of a generic collider experiment, JHEP 02 (2014) 057 [arXiv:1307.6346] [INSPIRE].

[57] M. Dobbs and J.B. Hansen, The HepMC C++ Monte Carlo event record for High Energy Physics, Comput. Phys. Commun. 134 (2001) 41 [INSPIRE].

[58] M. Cacciari, G.P. Salam and G. Soyez, The anti-k(t) jet clustering algorithm, JHEP 04 (2008) 063 [arXiv:0802.1189] [INSPIRE].

[59] C. Strege, G. Bertone, G.J. Besjes, S. Caron, R. Ruiz de Austri et al., Profile likelihood maps of a 15-dimensional MSSM, JHEP 09 (2014) 081 [arXiv:1405.0622] [INSPIRE].

[60] ATLAS collaboration, Search for direct production of charginos and neutralinos in events with three leptons and missing transverse momentum in $\sqrt{s}=8 \mathrm{TeV}$ pp collisions with the ATLAS detector, JHEP 04 (2014) 169 [arXiv: 1402.7029] [INSPIRE].

[61] ATLAS collaboration, Search for squarks and gluinos with the ATLAS detector in final states with jets and missing transverse momentum using $\sqrt{s}=8 \mathrm{TeV}$ proton-proton collision data, JHEP 09 (2014) 176 [arXiv:1405.7875] [INSPIRE].

[62] ATLAS collaboration, Search for strong production of supersymmetric particles in final states with missing transverse momentum and at least three b-jets at $\sqrt{s}=8 \mathrm{TeV}$ proton-proton collisions with the ATLAS detector, JHEP 10 (2014) 024 [arXiv:1407.0600] [INSPIRE].

[63] M.W. Cahill-Rowley, J.L. Hewett, A. Ismail and T.G. Rizzo, More energy, more searches, but the phenomenological MSSM lives on, Phys. Rev. D 88 (2013) 035002 [arXiv: 1211.1981] [INSPIRE].

[64] ATLAS collaboration, Search for squarks and gluinos in events with isolated leptons, jets and missing transverse momentum at $\sqrt{s}=8 \mathrm{TeV}$ with the ATLAS detector, ATLAS-CONF-2013-062 (2013).

[65] D. de Florian and J. Mazzitelli, Higgs Boson Pair Production at Next-to-Next-to-Leading Order in QCD, Phys. Rev. Lett. 111 (2013) 201801 [arXiv:1309.6594] [INSPIRE].

[66] J. Baglio et al., The measurement of the Higgs self-coupling at the LHC: theoretical status, JHEP 04 (2013) 151 [arXiv:1212.5581] [INSPIRE].

[67] M. Moretti, S. Moretti, F. Piccinini, R. Pittau and A.D. Polosa, Higgs boson self-couplings at the LHC as a probe of extended Higgs sectors, JHEP 02 (2005) 024 [hep-ph/0410334] [INSPIRE].

[68] T. Plehn and M. Rauch, The quartic Higgs coupling at hadron colliders, Phys. Rev. D 72 (2005) 053008 [hep-ph/0507321] [INSPIRE].

[69] B. Bhattacherjee and A. Choudhury, The role of MSSM heavy Higgs production in the self coupling measurement of the $125 \mathrm{GeV}$ Higgs boson at the LHC, Phys. Rev. D 91 (2015) 073015 [arXiv: 1407.6866] [inSPIRE]. 